\title{
ON THE STABILITY OF THE LINEAR DELAY DIFFERENTIAL AND DIFFERENCE EQUATIONS
}

\author{
A. ASHYRALYEV AND P. SOBOLEVSKII
}

Received 14 August 2001

We consider the initial-value problem for linear delay partial differential equations of the parabolic type. We give a sufficient condition for the stability of the solution of this initial-value problem. We present the stability estimates for the solutions of the first and second order accuracy difference schemes for approximately solving this initial-value problem. We obtain the stability estimates in Hölder norms for the solutions of the initial-value problem of the delay differential and difference equations of the parabolic type.

\section{Introduction}

Methods for numerical solutions of the delay ordinary differential equations have been studied extensively by many researchers (cf. $[1,2,3,4,6,7,8$, $9,10,12,13,14,15,16]$ and the references therein) and developed over the last two decades. In this literature, the linear delay equations was considering under the condition, such that in the case of the constant coefficients follows obviously from characteristic equations as the necessary condition. The stability of the solution of this initial-value problem and of the difference schemes for approximately solving the initial-value problem were obtained.

However, the theory and numerical solution of the delay partial differential equations have received less attention than the delay ordinary differential equations. In the present paper, we consider the initial-value problem for linear delay differential equations

$$
\begin{gathered}
\frac{d u(t)}{d t}+A u(t)=B(t) u(t-\omega), \quad t \geq 0, \\
u(t)=g(t), \quad-\omega \leq t \leq 0,
\end{gathered}
$$

in an arbitrary Banach space $E$ with unbounded linear operators $A$ and $B(t)$ in $E$ with dense domain $D(A) \subseteq D(B(t))$. Let $A$ be a strongly positive operator,

Copyright (C) 2001 Hindawi Publishing Corporation Abstract and Applied Analysis 6:5 (2001) 267-297

2000 Mathematics Subject Classification: 65J, 65M, 47D, 34K, 34G

URL: http://aaa.hindawi.com/volume-6/S1085337501000616.html 
that is, $-A$ is the generator of the analytic semigroup $\exp \{-t A\}(t \geq 0)$ of the linear bounded operators with exponentially decreasing norm when $t \rightarrow \infty$. This means the following estimates hold:

$$
\|\exp \{-t A\}\|_{E \mapsto E} \leq M e^{-\delta t}, \quad\|t A \exp \{-t A\}\|_{E \mapsto E} \leq M e^{-\delta t}, \quad t>0
$$

for some $M>0, \delta>0$ (see [11]).

A function $u(t)$ is called a solution of problem (1.1) if the following conditions are satisfied:

(i) The function $u(t)$ is continuously differentiable on the interval $[-\omega, \infty)$. The derivative at the endpoint $t=-\omega$ is understood as the appropriate unilateral derivative.

(ii) The function $u(t)$ belongs to $D(A)$ for all $t \in[-\omega, \infty)$, and the functions $A u(t)$ and $B(t) u(t)$ are continuous on the interval $[-\omega, \infty)$.

(iii) The function $u(t)$ satisfies the equation and the initial condition (1.1).

A solution $u(t)$ of the initial-value problem (1.1) is said to be stable if

$$
\|u(t)\|_{E} \leq \max _{-\omega \leq t \leq 0}\|g(t)\|_{E}
$$

for every $t,-\omega \leq t<\infty$. We are interested to study the stability of solutions of the initial-value problem under the assumption that

$$
\left\|B(t) A^{-1}\right\|_{E \mapsto E} \leq 1
$$

holds for every $t \geq 0$. We have not been able to obtain the estimate (1.3) in the arbitrary Banach space $E$. Nevertheless, we can establish the analog of estimates (1.3) where the space $E$ is replaced by the fractional spaces $E_{\alpha}(0<\alpha<1)$ under the more strong assumption than (1.4).

\section{The delay differential equation}

It is known that (cf. [17]) the strongly positive operator $A$ defines the fractional spaces $E_{\alpha}=E_{\alpha}(A, E)(0<\alpha<1)$ consisting of all $v \in E$ for which the following norms are finite:

$$
\|v\|_{E_{\alpha}}=\sup _{\lambda>0}\left\|\lambda^{1-\alpha} A \exp \{-\lambda A\} v\right\|_{E} .
$$

First, we consider problem (1.1) when

$$
A^{-1} B(t) x=B(t) A^{-1} x, \quad x \in D(A) .
$$


THEOREM 2.1. Assume that the condition

$$
\left\|B(t) A^{-1}\right\|_{E \mapsto E} \leq \frac{(1-\alpha)}{M 2^{2-\alpha}}
$$

holds for every $t \geq 0$. Then for every $t \geq 0$,

$$
\|u(t)\|_{E_{\alpha}} \leq \max _{-\omega \leq t \leq 0}\|g(t)\|_{E_{\alpha}} .
$$

Proof. Using the formula

$$
u(t)=\exp \{-t A\} g(0)+\int_{0}^{t} \exp \{-(t-s) A\} B(s) g(s-\omega) d s
$$

the semigroup property, condition (2.3), and the estimates (1.2) we have

$$
\begin{aligned}
\lambda^{1-\alpha} \| & A \exp \{-\lambda A\} u(t) \|_{E} \\
\leq & \lambda^{1-\alpha}\|A \exp \{-(\lambda+t) A\} g(0)\|_{E} \\
& +\lambda^{1-\alpha} \int_{0}^{t}\left\|A \exp \left\{-\frac{\lambda+t-s}{2} A\right\}\right\|_{E \rightarrow E}\left\|B(s) A^{-1}\right\|_{E \rightarrow E} \\
& \times\left\|A \exp \left\{-\frac{\lambda+t-s}{2} A\right\} g(s-\omega)\right\|_{E} d s \\
\leq & \frac{\lambda^{1-\alpha}}{(\lambda+t)^{1-\alpha}}\|g(0)\|_{E_{\alpha}}+\frac{1-\alpha}{M 2^{2-\alpha}} \int_{0}^{t} \frac{M \lambda^{1-\alpha} 2^{2-\alpha}}{(\lambda+t-s)^{2-\alpha}} d s \max _{0 \leq s \leq \omega}\|g(s-\omega)\|_{E_{\alpha}} \\
\leq & \max _{-\omega \leq t \leq 0}\|g(t)\|_{E_{\alpha}}
\end{aligned}
$$

for every $t, 0 \leq t \leq \omega$ and $\lambda, \lambda>0$. This shows that

$$
\|u(t)\|_{E_{\alpha}} \leq \max _{-\omega \leq t \leq 0}\|g(t)\|_{E_{\alpha}}
$$

for every $t, 0 \leq t \leq \omega$. Applying the mathematical induction, one can easily show that it is true for every $t$. Namely, assume that inequality (2.4) is true for $t,(n-1) \omega \leq t \leq n \omega, n=1,2,3, \ldots$. Using the formula

$$
u(t)=\exp \{-(t-n \omega) A\} u(n \omega)+\int_{n \omega}^{t} \exp \{-(t-s) A\} B(s) u(s-\omega) d s
$$


the semigroup property, condition (2.3), and the estimates (1.2) we have

$$
\begin{aligned}
\lambda^{1-\alpha} \| & A \exp \{-\lambda A\} u(t) \|_{E} \\
\leq & \lambda^{1-\alpha}\|A \exp \{-(\lambda+t-n \omega) A\} u(n \omega)\|_{E} \\
& +\lambda^{1-\alpha} \int_{n \omega}^{t}\left\|A \exp \left\{-\frac{\lambda+t-s}{2} A\right\}\right\|_{E \rightarrow E}\left\|B(s) A^{-1}\right\|_{E \rightarrow E} \\
& \times\left\|A \exp \left\{-\frac{\lambda+t-s}{2} A\right\} u(s-\omega)\right\|_{E} d s \\
\leq & \frac{\lambda^{1-\alpha}}{(\lambda+t-n \omega)^{1-\alpha}}\|u(n \omega)\|_{E_{\alpha}}+\frac{1-\alpha}{M 2^{2-\alpha}} \int_{n \omega}^{t} \frac{M \lambda^{1-\alpha} 2^{2-\alpha}}{(\lambda+t-s)^{2-\alpha}} d s \\
& \times \max _{n \omega \leq s \leq(n+1) \omega}\|u(s-\omega)\|_{E_{\alpha}} \\
\leq & \max _{-(n-1) \omega \leq t \leq n \omega}\|u(t)\|_{E_{\alpha}}
\end{aligned}
$$

for every $t, n \omega \leq t \leq(n+1) \omega, n=1,2,3, \ldots$ and $\lambda, \lambda>0$. This shows that

$$
\|u(t)\|_{E_{\alpha}} \leq \max _{-\omega \leq t \leq 0}\|g(t)\|_{E_{\alpha}}
$$

for every $t, n \omega \leq t \leq(n+1) \omega, n=1,2,3, \ldots$. This result completes the proof of Theorem 2.1.

Now, we consider problem (1.1) when

$$
A^{-1} B(t) x \neq B(t) A^{-1} x, \quad x \in D(A) .
$$

Note that (see [11]) $A$ is a strongly positive operator in a Banach space $E$ if and only if its spectrum $\sigma(A)$ lies in the interior sector of angle $\varphi, 0<2 \varphi<\pi$, symmetric with respect to the real axis, and if on the edges of this sector, $S_{1}=[z=\rho \exp (i \varphi): 0 \leq \rho<\infty]$ and $S_{2}=[z=\rho \exp (-i \varphi): 0 \leq \rho<\infty]$, and outside it the resolvent $(z-A)^{-1}$ is subject to the bound

$$
\left\|(z-A)^{-1}\right\|_{E \rightarrow E} \leq \frac{M_{1}}{1+|z|}
$$

for some $M_{1}>0$. Throughout, the operator $(z I-A)^{-1}$ will be written $(z-A)^{-1}$. First of all we prove a lemma that will be needed in the sequel.

Lemma 2.2. For any $z$ on the edges of the sector, $S_{1}=[z=\rho \exp (i \varphi): 0 \leq \rho<$ $\infty]$, and $S_{2}=[z=\rho \exp (-i \varphi): 0 \leq \rho<\infty]$, and outside it the estimate

$$
\left\|A(z-A)^{-1} \varphi\right\|_{E} \leq \frac{M_{1}^{\alpha} M^{\alpha}\left(1+M_{1}\right)^{1-\alpha} 2^{(2-\alpha) \alpha}}{\alpha(1-\alpha)(1+|z|)^{\alpha}}\|\varphi\|_{E_{\alpha}}
$$


holds. Here and in the future $M$ and $M_{1}$ are some constants of the estimates (1.2) and (2.12).

Proof. Applying the formula (see [11])

$$
\varphi=\int_{0}^{\infty} A \exp \{-\tau A\} \varphi d \tau,
$$

and using the semigroup property we obtain

$$
\begin{aligned}
A(z-A)^{-1} \varphi= & \int_{0}^{N} A(z-A)^{-1} A \exp \{-\tau A\} \varphi d \tau \\
& +\int_{N}^{\infty}(z-A)^{-1} A \exp \left\{-\frac{\tau}{2} A\right\} A \exp \left\{-\frac{\tau}{2} A\right\} \varphi d \tau .
\end{aligned}
$$

Using the estimates (1.2), (2.12), and the definition of the spaces $E_{a}$, we obtain

$$
\begin{aligned}
\left\|A(z-A)^{-1} \varphi\right\|_{E} \leq & \int_{0}^{N}\left\|A(z-A)^{-1}\right\|_{E \rightarrow E}\|A \exp \{-\tau A\} \varphi\|_{E} d \tau \\
& +\int_{N}^{\infty}\left\|(z-A)^{-1}\right\|_{E \mapsto E}\left\|A \exp \left\{-\frac{\tau}{2} A\right\}\right\|_{E \mapsto E} \\
& \times\left\|A \exp \left\{-\frac{\tau}{2} A\right\} \varphi\right\|_{E} d \tau \\
\leq & \left(\int_{0}^{N}\left(1+M_{1}\right) \tau^{\alpha-1} d \tau+\int_{N}^{\infty} \frac{M M_{1} 2^{2-\alpha} \tau^{\alpha-2}}{|z|+1} d \tau\right)\|\varphi\|_{E_{\alpha}} \\
= & \psi(N)\|\varphi\|_{E_{\alpha}} .
\end{aligned}
$$

Here

$$
\psi(N)=\left(1+M_{1}\right) \frac{N^{\alpha}}{\alpha}+\frac{M M_{1} 2^{2-\alpha} N^{\alpha-1}}{(|z|+1)(1-\alpha)} .
$$

Taking the minimum of the function $\psi(N)$ over all $N, 0<N<\infty$, we obtain Lemma 2.2.

Lemma 2.3. For all $\tau>0$ the following estimate holds:

$$
\begin{aligned}
\| A^{-1} & {[A \exp \{-\tau A\} B(s)-B(s) A \exp \{-\tau A\}] \varphi \|_{E} } \\
& \leq \frac{e(\alpha+1) M^{\alpha} M_{1}^{1+\alpha}\left(1+2 M_{1}\right)\left(1+M_{1}\right)^{1-\alpha} 2^{(2-\alpha) \alpha}\|Q\|_{E \mapsto E}\|\varphi\|_{E_{\alpha}}}{\tau^{1-\alpha} \pi \alpha^{2}(1-\alpha)} .
\end{aligned}
$$

Here $Q=\overline{A^{-1}(A B(s)-B(s) A) A^{-1}}$. 
272 Delay differential and difference equations

Proof. Applying the Cauchy-Riesz formula, we obtain the representation

$$
\begin{aligned}
A \exp \{-\tau A\} \varphi= & \frac{1}{2 \pi i} \int_{S_{1} \cup S_{2}} \exp \{-\tau z\} A(z-A)^{-1} d z \varphi \\
= & -\frac{1}{2 \pi i} \int_{S_{1} \cup S_{2}} \exp \{-\tau z\} d z \varphi \\
& +\frac{1}{2 \pi i} \int_{S_{1} \cup S_{2}} \exp \{-\tau z\} z(z-A)^{-1} d z \varphi
\end{aligned}
$$

for every $\tau, \tau>0$. From that it follows that

$$
\begin{aligned}
A^{-1} & {[A \exp \{-\tau A\} B(s)-B(s) A \exp \{-\tau A\}] \varphi } \\
& =\frac{1}{2 \pi i} \int_{S_{1} \cup S_{2}} \exp \{-\tau z\} z A^{-1}\left[(z-A)^{-1} B(s)-B(s)(z-A)^{-1}\right] d z \varphi \\
& =\frac{1}{2 \pi i} \int_{S_{1} \cup S_{2}} \exp \{-\tau z\} z(z-A)^{-1} Q A(z-A)^{-1} d z \varphi .
\end{aligned}
$$

Making the substitution $z=-1 / \tau+i y,-\infty<y<\infty$ and using integration by parts, we can write the formula

$$
\begin{aligned}
& A^{-1}[A \exp \{-\tau A\} B(s)-B(s) A \exp \{-\tau A\}] \varphi \\
&=\frac{e}{2 \pi} \int_{-\infty}^{\infty} \exp (-\tau i y)\left(-\frac{1}{\tau}+i y\right)\left(-\frac{1}{\tau}+i y-A\right)^{-1} Q A \\
& \quad \times\left(-\frac{1}{\tau}+i y-A\right)^{-1} d y \varphi \\
&=\frac{e}{2 \pi \tau} \int_{-\infty}^{\infty} \exp (-\tau i y)\left(-\frac{1}{\tau}+i y-A\right)^{-1} \\
& \times\left[Q-\left(-\frac{1}{\tau}+i y\right)\left(-\frac{1}{\tau}+i y-A\right)^{-1} Q\right. \\
& \times A\left(-\frac{1}{\tau}+i y-A\right)^{-1} d y \varphi .
\end{aligned}
$$


By Lemma 2.2 and performing the change of variable $\tau y=x$, and using the estimate (2.12), we obtain

$$
\begin{aligned}
\| A^{-1}[ & A \exp \{-\tau A\} B(s)-B(s) A \exp \{-\tau A\}] \varphi \|_{E} \\
\leq & \frac{e}{2 \pi \tau} \int_{-\infty}^{\infty}|\exp (-\tau i y)|\left\|\left(-\frac{1}{\tau}+i y-A\right)^{-1}\right\|_{E \mapsto E} \\
& \times\left(1+2 M_{1}\right)\|Q\|_{E \mapsto E}\left\|A\left(-\frac{1}{\tau}+i y-A\right)^{-1} d y \varphi\right\|_{E} \\
\leq & \frac{e}{2 \pi \tau} \int_{-\infty}^{\infty} \frac{d y}{\left(1 / \tau^{2}+y^{2}\right)^{(1+\alpha) / 2}} \\
& \times \frac{\left(1+2 M_{1}\right)\|Q\|_{E \mapsto E} M_{1}^{1+\alpha} M^{\alpha}\left(1+M_{1}\right)^{1-\alpha} 2^{(2-\alpha) \alpha}\|\varphi\|_{E_{\alpha}}}{\alpha(1-\alpha)} \\
= & \frac{e}{\pi \tau^{1-\alpha}} \int_{0}^{\infty} \frac{d x}{\left(1+x^{2}\right)^{(1+\alpha) / 2}} \\
& \times \frac{\left(1+2 M_{1}\right)\|Q\|_{E \mapsto E} M_{1}^{1+\alpha} M^{\alpha}\left(1+M_{1}\right)^{1-\alpha} 2^{(2-\alpha) \alpha}\|\varphi\|_{E_{\alpha}}}{\alpha(1-\alpha)} \\
\leq & \frac{e(1+\alpha)\left(1+2 M_{1}\right)\|Q\|_{E \mapsto E} M_{1}^{1+\alpha} M^{\alpha}\left(1+M_{1}\right)^{1-\alpha} 2^{(2-\alpha) \alpha}\|\varphi\|_{E_{\alpha}}}{\pi \tau^{1-\alpha} \alpha^{2}(1-\alpha)} .
\end{aligned}
$$

Lemma 2.3 is thus proved.

Suppose that

$$
\begin{aligned}
& \left\|\overline{A^{-1}(A B(t)-B(t) A) A^{-1}}\right\|_{E \mapsto E} \\
& \leq \frac{\pi(1-\alpha)^{2} \alpha^{2} \epsilon}{e M^{1+\alpha} M_{1}^{1+\alpha}\left(1+2 M_{1}\right)\left(1+M_{1}\right)^{1-\alpha} 2^{2+\alpha-\alpha^{2}}(1+\alpha)}
\end{aligned}
$$

holds for every $t \geq 0$. Here and in the future $\epsilon$ is some constant, $0 \leq \epsilon \leq 1$.

Theorem 2.4. Assume that the condition

$$
\left\|\overline{A^{-1} B(t)}\right\|_{E \mapsto E} \leq \frac{(1-\alpha)(1-\epsilon)}{M 2^{2-\alpha}}
$$

holds for every $t \geq 0$. Then for every $t \geq 0$ the estimate (2.4) holds. 
274 Delay differential and difference equations

Proof. Using formula (2.5) we have

$$
\begin{aligned}
\lambda^{1-\alpha} A & \exp \{-\lambda A\} u(t) \\
= & \lambda^{1-\alpha} A \exp \{-(\lambda+t) A\} g(0) \\
+\lambda^{1-\alpha} \int_{0}^{t} \exp \left\{-\frac{\lambda+t-s}{2} A\right\} B(s) & \times A \exp \left\{-\frac{\lambda+t-s}{2} A\right\} g(s-\omega) d s \\
+\lambda^{1-\alpha} \int_{0}^{t} \exp \left\{-\frac{\lambda+t-s}{2} A\right\} & \times\left[A \exp \left\{-\frac{\lambda+t-s}{2} A\right\} B(s)\right. \\
& \left.\quad-B(s) A \exp \left\{-\frac{\lambda+t-s}{2} A\right\}\right] g(s-\omega) d s \\
=I_{1}+I_{2}+I_{3} . &
\end{aligned}
$$

Using the estimates (1.2), (2.12), and condition (2.24) we have

$$
\begin{aligned}
&\left\|I_{1}\right\|_{E}=\lambda^{1-\alpha}\|A \exp \{-(\lambda+t) A\} g(0)\|_{E} \\
& \leq \frac{\lambda^{1-\alpha}}{(\lambda+t)^{1-\alpha}}\|g(0)\|_{E_{\alpha}} \leq \frac{\lambda^{1-\alpha}}{(\lambda+t)^{1-\alpha}} \max _{-\omega \leq t \leq 0}\|g(t)\|_{E_{\alpha}} \\
&\left\|I_{2}\right\|_{E} \leq \lambda^{1-\alpha} \int_{0}^{t}\left\|A \exp \left\{-\frac{\lambda+t-s}{2} A\right\}\right\|_{E \mapsto E}\left\|\overline{A^{-1} B(s)}\right\|_{E \mapsto E} \\
& \quad \times\left\|A \exp \left\{-\frac{\lambda+t-s}{2} A\right\} g(s-\omega)\right\|_{E} d s \\
& \leq \max _{0 \leq t \leq \omega}\left\|\frac{A^{-1} B(t)}{2}\right\|_{E \mapsto E} \int_{0}^{t} \frac{M \lambda^{1-\alpha} 2^{2-\alpha}}{(\lambda+t-s)^{2-\alpha}} d s \max _{0 \leq s \leq \omega}\|g(s-\omega)\|_{E_{\alpha}} \\
& \leq \max _{-\omega \leq t \leq 0}\|g(t)\|_{E_{\alpha}}\left(1-\frac{\lambda^{1-\alpha}}{(\lambda+t)^{1-\alpha}}\right)(1-\epsilon)
\end{aligned}
$$


for every $t, 0 \leq t \leq \omega$ and $\lambda, \lambda>0$. Now we estimate $I_{3}$. By Lemma 2.3 and using the estimate (2.23), we obtain

$$
\begin{aligned}
& \left\|I_{3}\right\|_{E} \leq \lambda^{1-\alpha} \int_{0}^{t}\left\|A \exp \left\{-\frac{\lambda+t-s}{2} A\right\}\right\|_{E \mapsto E} \\
& \times \| A^{-1}\left[A \exp \left\{-\frac{\lambda+t-s}{2} A\right\} B(s)-B(s)\right. \\
& \left.\times A \exp \left\{-\frac{\lambda+t-s}{2} A\right\}\right] g(s-\omega) \|_{E} d s \\
& \leq \lambda^{1-\alpha} e(1+\alpha) M^{1+\alpha} M_{1}^{1+\alpha}\left(1+2 M_{1}\right)\left(1+M_{1}\right)^{1-\alpha} 2^{(2-\alpha) \alpha} \\
& \times \int_{0}^{t} \frac{\left\|\overline{A^{-1}(A B(s)-B(s) A) A^{-1}}\right\|_{E \mapsto E} 2^{2-\alpha}\|g(s-\omega)\|_{E_{\alpha}} d s}{(\lambda+t-s)^{2-\alpha} \pi \alpha^{2}(1-\alpha)} \\
& \leq \max _{0 \leq s \leq \omega}\left\|\overline{A^{-1}(A B(s)-B(s) A) A^{-1}}\right\|_{E \mapsto E} \\
& \times \int_{0}^{t} \frac{e(1+\alpha) M^{1+\alpha} M_{1}^{1+\alpha}\left(1+2 M_{1}\right)\left(1+M_{1}\right)^{1-\alpha} 2^{(2-\alpha) \alpha} 2^{2-\alpha} d s}{(\lambda+t-s)^{2-\alpha} \pi \alpha^{2}(1-\alpha)} \\
& \times \max _{-\omega \leq t \leq 0}\|g(t)\|_{E_{\alpha}} \\
& \leq \max _{-\omega \leq t \leq 0}\|g(t)\|_{E_{\alpha}}\left(1-\frac{\lambda^{1-\alpha}}{(\lambda+t)^{1-\alpha}}\right) \epsilon
\end{aligned}
$$

for every $t, 0 \leq t \leq \omega$ and $\lambda, \lambda>0$. Using the triangle inequality we obtain

$$
\lambda^{1-\alpha}\|A \exp \{-\lambda A\} u(t)\|_{E} \leq \max _{-\omega \leq t \leq 0}\|g(t)\|_{E_{\alpha}}
$$

for every $t, 0 \leq t \leq \omega$ and $\lambda, \lambda>0$. This shows that

$$
\|u(t)\|_{E_{\alpha}} \leq \max _{-\omega \leq t \leq 0}\|g(t)\|_{E_{\alpha}}
$$

for every $t, 0 \leq t \leq \omega$. Applying the mathematical induction, one can easily show that it is true for every $t$. Namely, assume that inequality (2.4) is true for every $t,(n-1) \omega \leq t \leq n \omega, n=1,2,3, \ldots$ Therefore, using formula (2.24) 
we have

$$
\begin{aligned}
\lambda^{1-\alpha} A \exp \{-\lambda A\} u(t) & \\
= & \lambda^{1-\alpha} A \exp \{-(\lambda+t-n \omega) A\} u(n \omega) \\
& +\lambda^{1-\alpha} \int_{n \omega}^{t} \exp \left\{-\frac{\lambda+t-s}{2} A\right\} B(s) A \exp \left\{-\frac{\lambda+t-s}{2} A\right\} u(s-\omega) d s \\
& +\lambda^{1-\alpha} \int_{n \omega}^{t} \exp \left\{-\frac{\lambda+t-s}{2} A\right\}\left[A \exp \left\{-\frac{\lambda+t-s}{2} A\right\} B(s)\right. \\
& \left.\quad-B(s) A \exp \left\{-\frac{\lambda+t-s}{2} A\right\}\right] \\
& \times u(s-\omega) d s \\
= & I_{1 n}+I_{2 n}+I_{3 n} .
\end{aligned}
$$

Using the estimates (1.2), (2.12), and condition (2.24), we have

$$
\begin{aligned}
\left\|I_{1 n}\right\|_{E} & =\lambda^{1-\alpha}\|A \exp \{-(\lambda+t-n \omega) A\} u(n \omega)\|_{E} \\
& \leq \frac{\lambda^{1-\alpha}}{(\lambda+t-n \omega)^{1-\alpha}}\|u(n \omega)\|_{E_{\alpha}} \leq \frac{\lambda^{1-\alpha}}{(\lambda+t-n \omega)^{1-\alpha}} \max _{-\omega \leq t \leq 0}\|g(t)\|_{E_{\alpha}}, \\
\left\|I_{2 n}\right\|_{E} \leq & \lambda^{1-\alpha} \int_{n \omega}^{t}\left\|A \exp \left\{-\frac{\lambda+t-s}{2} A\right\}\right\|_{E \mapsto E}\left\|\overline{A^{-1} B(s)}\right\|_{E \mapsto E} \\
& \times\left\|A \exp \left\{-\frac{\lambda+t-s}{2} A\right\} u(s-\omega)\right\|_{E} d s \\
\leq & \max _{n \omega \leq t \leq(n+1) \omega}\left\|\overline{A^{-1} B(t)}\right\|_{E \mapsto E} \int_{n \omega}^{t} \frac{M \lambda^{1-\alpha} 2^{2-\alpha}}{(\lambda+t-s)^{2-\alpha}} d s \\
& \times \max _{n \omega \leq s \leq(n+1) \omega}\|u(s-\omega)\|_{E_{\alpha}} \\
\leq & \max _{-\omega \leq t \leq 0}\|g(t)\| E_{E_{\alpha}}\left(1-\frac{\lambda^{1-\alpha}}{(\lambda+t)^{1-\alpha}}\right)(1-\epsilon)
\end{aligned}
$$


for every $t, n \omega \leq t \leq(n+1) \omega$ and $\lambda, \lambda>0$. Now we estimate $I_{3 n}$. By Lemma 2.3 and using the estimate (2.23), we obtain

$$
\begin{aligned}
\left\|I_{3 n}\right\|_{E} \leq & \lambda^{1-\alpha} \int_{n \omega}^{t}\left\|A \exp \left\{-\frac{\lambda+t-s}{2} A\right\}\right\|_{E \mapsto E} \\
\times \| A^{-1}\left[A \exp \left\{-\frac{\lambda+t-s}{2} A\right\} B(s)\right. & \left.-B(s) A \exp \left\{-\frac{\lambda+t-s}{2} A\right\}\right] u(s-\omega) \|_{E} d s \\
\leq & \lambda^{1-\alpha} e(1+\alpha) M^{1+\alpha} M_{1}^{1+\alpha}\left(1+2 M_{1}\right)\left(1+M_{1}\right)^{1-\alpha} 2^{(2-\alpha) \alpha} \\
& \times \int_{n \omega}^{t} \frac{\| A^{-1}(A B(s)-B(s) A) A^{-1}}{} \frac{(\lambda+t-s)^{2-\alpha} \pi \alpha^{2}(1-\alpha)}{\left\|2^{2-\alpha}\right\| u(s-\omega) \|_{E_{\alpha}} d s} \\
\leq & \max _{n \omega \leq s \leq(n+1) \omega}\left\|A^{-1}(A B(s)-B(s) A) A^{-1}\right\|_{E \mapsto E} \\
& \times \int_{n \omega}^{t} \frac{e(1+\alpha) M^{1+\alpha} M_{1}^{1+\alpha}\left(1+2 M_{1}\right)\left(1+M_{1}\right)^{1-\alpha} 2^{(2-\alpha) \alpha} 2^{2-\alpha} d s}{(\lambda+t-s)^{2-\alpha} \pi \alpha^{2}(1-\alpha)} \\
& \times \max _{n \omega \leq t \leq(n+1) \omega}\|u(s-\omega)\|_{E_{\alpha}} \\
\leq & \max _{-\omega \leq t \leq 0}\|g(t)\| E_{\alpha}\left(1-\frac{\lambda^{1-\alpha}}{(\lambda+t)^{1-\alpha}}\right) \epsilon
\end{aligned}
$$

for every $t, n \omega \leq t \leq(n+1) \omega$ and $\lambda, \lambda>0$. Using the triangle inequality we obtain

$$
\lambda^{1-\alpha}\|A \exp \{-\lambda A\} u(t)\|_{E} \leq \max _{-\omega \leq t \leq 0}\|g(t)\|_{E_{\alpha}}
$$

for every $t, n \omega \leq t \leq(n+1) \omega, n=1,2,3, \ldots$, and $\lambda, \lambda>0$. This shows that

$$
\|u(t)\|_{E_{\alpha}} \leq \max _{-\omega \leq t \leq 0}\|g(t)\|_{E_{\alpha}}
$$

for every $t, n \omega \leq t \leq(n+1) \omega, n=1,2,3, \ldots$. This result completes the proof of Theorem 2.4.

\section{The delay difference equation}

Using the first and second order accuracy implicit difference schemes for differential equations without delay (cf. [5]), we have the following approximate 
solution for the initial-value problem (1.1)

$$
\begin{gathered}
\frac{1}{\tau}\left(u_{k}-u_{k-1}\right)+A u_{k}=B_{k} u_{k-N}, \\
B_{k}=B\left(t_{k}\right), \quad t_{k}=k \tau, \quad 1 \leq k, \quad N \tau=\omega, \\
u_{k}=g\left(t_{k}\right), \quad t_{k}=k \tau,-N \leq k \leq 0 ; \\
\frac{1}{\tau}\left(u_{k}-u_{k-1}\right)+\left(A+\frac{1}{2} \tau A^{2}\right) u_{k}=\frac{1}{2}\left(I+\frac{\tau}{2} A\right) B_{k}\left(u_{k-N}+u_{k-N-1}\right), \\
B_{k}=B\left(t_{k}-\frac{\tau}{2}\right), \quad t_{k}=k \tau, \quad 1 \leq k, \\
u_{k}=g\left(t_{k}\right), \quad t_{k}=k \tau,-N \leq k \leq 0 .
\end{gathered}
$$

TheOREM 3.1. Assume that all the conditions of Theorem 2.1 are satisfied. Then for the solution of difference scheme (3.1) the estimate

$$
\left\|u_{k}\right\|_{E_{\alpha}} \leq \max _{-N \leq k \leq 0}\left\|g\left(t_{k}\right)\right\|_{E_{\alpha}}
$$

holds for any $k \geq 1$.

Proof. Consider $1 \leq k \leq N$. In this case

$$
u_{k}=R^{k} g(0)+\sum_{j=1}^{k} R^{k-j+1} g\left(t_{j-N}\right) \tau
$$

where $R=(1+\tau A)^{-1}$. Using the formula (see [5, 11])

$$
(I+\tau A)^{-k}=\frac{1}{(k-1) !} \int_{0}^{\infty} t^{k-1} \exp (-t) \exp (-\tau t A) d t, \quad k \geq 1,
$$

and condition (2.3), we have

$$
\begin{aligned}
& \lambda^{1-\alpha} \| A \exp \{-\lambda A\} u_{k} \|_{E} \\
& \leq \lambda^{1-\alpha} \frac{1}{(k-1) !} \int_{0}^{\infty} t^{k-1} \exp (-t)\|A \exp \{-(\tau t+\lambda) A\} g(0)\|_{E} \\
&+ \lambda^{1-\alpha} \sum_{j=1}^{k} \tau \frac{1}{(k-j) !} \\
& \quad \times \int_{0}^{\infty} t^{k-j} \exp (-t)\left\|A \exp \left\{-\frac{(\tau t+\lambda) A}{2}\right\}\right\|_{E \mapsto E}\left\|B_{j} A^{-1}\right\|_{E \mapsto E} \\
& \quad \times\left\|A \exp \left\{-\frac{(\tau t+\lambda) A}{2}\right\} g\left(t_{j-N}\right)\right\|_{E}
\end{aligned}
$$




$$
\begin{aligned}
\leq & \lambda^{1-\alpha} \frac{1}{(k-1) !} \int_{0}^{\infty} t^{k-1} \exp (-t) \frac{d t}{(t \tau+\lambda)^{1-\alpha}}\|g(0)\|_{E_{\alpha}} \\
& +\lambda^{1-\alpha} \frac{1-\alpha}{M 2^{2-\alpha}} \sum_{j=1}^{k} \tau \frac{1}{(k-j) !} \\
& \times \int_{0}^{\infty} t^{k-j} \exp (-t) \frac{M 2^{2-\alpha} d t}{(t \tau+\lambda)^{2-\alpha}}\left\|g\left(t_{j-N}\right)\right\|_{E_{\alpha}} \\
\leq & J \max _{-N \leq k \leq 0}\left\|g\left(t_{k}\right)\right\|_{E_{\alpha}},
\end{aligned}
$$

where

$$
\begin{aligned}
J= & \lambda^{1-\alpha} \frac{1}{(k-1) !} \int_{0}^{\infty} t^{k-1} \exp (-t) \frac{d t}{(t \tau+\lambda)^{1-\alpha}} \\
& +\lambda^{1-\alpha}(1-\alpha) \sum_{j=1}^{k} \tau \frac{1}{(k-j) !} \int_{0}^{\infty} t^{k-j} \exp (-t) \frac{d t}{(t \tau+\lambda)^{2-\alpha}} .
\end{aligned}
$$

We will calculate $J$. Making the substitution $k-j=m$ and using Taylor's formula and integration by parts, we obtain

$$
\begin{aligned}
J= & \lambda^{1-\alpha} \frac{1}{(k-1) !} \int_{0}^{\infty} t^{k-1} \exp (-t) \frac{d t}{(t \tau+\lambda)^{1-\alpha}} \\
& +\lambda^{1-\alpha}(1-\alpha) \sum_{m=0}^{k-1} \frac{1}{m !} \int_{0}^{\infty} t^{m} \exp (-t) \frac{d t}{(t \tau+\lambda)^{2-\alpha}} \\
= & \lambda^{1-\alpha} \frac{1}{(k-1) !} \int_{0}^{\infty} t^{k-1} \exp (-t) \frac{d t}{(t \tau+\lambda)^{1-\alpha}} \\
& +\lambda^{1-\alpha}(1-\alpha) \int_{0}^{\infty} \exp (t)\left[1-\int_{0}^{t} \frac{\xi^{k-1} \exp (-\xi) d \xi}{(k-1) !}\right] \exp (-t) \frac{d t}{(t \tau+\lambda)^{2-\alpha}} \\
= & \lambda^{1-\alpha} \frac{1}{(k-1) !} \int_{0}^{\infty} t^{k-1} \exp (-t) \frac{d t}{(t \tau+\lambda)^{1-\alpha}}+1 \\
& -\lambda^{1-\alpha} \frac{1}{(k-1) !} \int_{0}^{\infty} t^{k-1} \exp (-t) \frac{d t}{(t \tau+\lambda)^{1-\alpha}} \\
= & 1 .
\end{aligned}
$$

Therefore,

$$
\lambda^{1-\alpha}\left\|A \exp \{-\lambda A\} u_{k}\right\|_{E} \leq \max _{-N \leq k \leq 0}\left\|g\left(t_{k}\right)\right\|_{E_{\alpha}}
$$


for every $k, 1 \leq k \leq N$ and $\lambda, \lambda>0$. This shows that

$$
\left\|u_{k}\right\|_{E_{\alpha}} \leq \max _{-N \leq k \leq 0}\left\|g\left(t_{k}\right)\right\|_{E_{\alpha}}
$$

for every $k, 1 \leq k \leq N$. Assume that the estimate (3.2) is true for $k$, where $(n-1) N \leq k \leq n N, n=1,2,3, \ldots$. Then for any $k$, with $n N \leq k \leq(n+1) N$ using formula (3.5) and

$$
u_{k}=R^{k-n N} u_{n N}+\sum_{j=n N+1}^{k} \tau R^{k-j+1} B_{j} u_{j-N}
$$

and the estimates (1.2) and condition (2.3), we have

$$
\begin{aligned}
\lambda^{1-\alpha} \| & A \exp \{-\lambda A\} u_{k} \|_{E} \\
\leq & \lambda^{1-\alpha} \frac{1}{(k-n N-1) !} \int_{0}^{\infty} t^{k-n N-1} \exp (-t)\left\|A \exp (-(\tau t+\lambda) A) u_{n N}\right\|_{E} \\
& +\lambda^{1-\alpha} \sum_{j=n N+1}^{k} \tau \frac{1}{(k-j) !} \int_{0}^{\infty} t^{k-j} \exp (-t)\left\|A \exp \left\{-\frac{(\tau t+\lambda) A}{2}\right\}\right\|_{E \rightarrow E} \\
& \times\left\|B_{j} A^{-1}\right\|\left\|_{E \rightarrow E} \times\right\| \exp \left\{-\frac{(\tau t+\lambda) A}{2}\right\} u_{j-N} \|_{E} \\
\leq & \lambda^{1-\alpha} \frac{1}{(k-n N-1) !} \int_{0}^{\infty} t^{k-n N-1} \exp (-t) \frac{d t}{(t \tau+\lambda)^{1-\alpha}}\left\|u_{n N}\right\|_{E_{\alpha}} \\
& +\lambda^{1-\alpha} \frac{1-\alpha}{M 2^{2-\alpha}} \sum_{j=n N+1}^{k} \tau \frac{1}{(k-j) !} \int_{0}^{\infty} t^{k-j} \exp (-t) \frac{M 2^{2-\alpha} d t}{(t \tau+\lambda)^{2-\alpha}}\left\|u_{j-N}\right\|_{E_{\alpha}} \\
\leq & J_{n} \max _{(n-1) N \leq k \leq n N}\left\|u_{k}\right\|_{E_{\alpha}},
\end{aligned}
$$

where

$$
\begin{aligned}
J_{n}= & \lambda^{1-\alpha} \frac{1}{(k-n N-1) !} \int_{0}^{\infty} t^{k-n N-1} \exp (-t) \frac{d t}{(t \tau+\lambda)^{1-\alpha}} \\
& +\lambda^{1-\alpha}(1-\alpha) \sum_{j=n N+1}^{k} \tau \frac{1}{(k-j) !} \int_{0}^{\infty} t^{k-j} \exp (-t) \frac{d t}{(t \tau+\lambda)^{2-\alpha}}
\end{aligned}
$$


We will calculate $J_{n}$. Making the substitution $k-j=m$ and using Taylor's formula and integration by parts, we obtain

$$
\begin{aligned}
J_{n}= & \lambda^{1-\alpha} \frac{1}{(k-n N-1) !} \int_{0}^{\infty} t^{k-n N-1} \exp (-t) \frac{d t}{(t \tau+\lambda)^{1-\alpha}} \\
& +\lambda^{1-\alpha}(1-\alpha) \sum_{m=0}^{k-n N-1} \tau \frac{1}{m !} \int_{0}^{\infty} t^{m} \exp (-t) \frac{d t}{(t \tau+\lambda)^{2-\alpha}} \\
= & \lambda^{1-\alpha} \frac{1}{(k-n N-1) !} \int_{0}^{\infty} t^{k-n N-1} \exp (-t) \frac{d t}{(t \tau+\lambda)^{1-\alpha}} \\
& +\lambda^{1-\alpha}(1-\alpha) \int_{0}^{\infty} \exp (t) \\
= & \lambda^{1-\alpha} \frac{1}{(k-n N-1) !} \int_{0}^{\infty} t^{k-n N-1} \exp (-t) \frac{d t}{(t \tau+\lambda)^{1-\alpha}} \\
& \left.+1-\lambda^{1-\alpha} \frac{1}{(k-n N-1) !} \int_{0}^{\xi^{k-n N-1} \exp (-\xi) d \xi}\right] \frac{\exp (-t) \frac{d t}{(t \tau+\lambda)^{2-\alpha}}}{(k-n N-1) !} \\
= & 1 .
\end{aligned}
$$

Therefore

$$
\lambda^{1-\alpha}\left\|A \exp \{-\lambda A\} u_{k}\right\|_{E} \leq \max _{-N \leq k \leq 0}\left\|g\left(t_{k}\right)\right\|_{E_{\alpha}}
$$

for every $k, n N \leq k \leq(n+1) N, n=1,2,3, \ldots$, and $\lambda, \lambda>0$. This shows that

$$
\left\|u_{k}\right\|_{E_{\alpha}} \leq \max _{-\omega \leq k \leq 0}\left\|g\left(t_{k}\right)\right\|_{E_{\alpha}}
$$

for every $k, n N \leq k \leq(n+1) N, n=1,2,3, \ldots$ This result completes the proof of Theorem 3.1.

ThEOREM 3.2. Assume that all the conditions of Theorem 2.4 are satisfied. Then for the solution of the difference scheme (3.1), the estimate (3.3) holds for any $k \geq 1$.

Proof. Consider $1 \leq k \leq N$. Using formula (3.5) we have 
Delay differential and difference equations

$$
\begin{aligned}
& \lambda^{1-\alpha} A \exp \{-\lambda A\} u_{k}=\lambda^{1-\alpha} \frac{1}{(k-1) !} \int_{0}^{\infty} t^{k-1} \exp (-t) A \exp (-(\tau t+\lambda) A) g(0) \\
& +\lambda^{1-\alpha} \sum_{j=1}^{k} \tau \frac{1}{(k-j) !} \int_{0}^{\infty} t^{k-j} \exp (-t) \exp \left\{-\frac{\tau t+\lambda}{2} A\right\} \\
& \quad \times B_{j} A \exp \left\{-\frac{\tau t+\lambda}{2} A\right\} g\left(t_{j-N}\right) \\
& +\lambda^{1-\alpha} \sum_{j=1}^{k} \tau \frac{1}{(k-j) !} \\
& \times \int_{0}^{\infty} t^{k-j} \exp (-t) A \exp \left\{-\frac{\tau t+\lambda}{2} A\right\} A^{-1} \\
& \quad \times\left[\exp \left\{-\frac{\tau t+\lambda}{2} A\right\} A B_{j}-B_{j} A \exp \left\{-\frac{\tau t+\lambda}{2} A\right\}\right] g\left(t_{j-N}\right) \\
& =T_{1}+T_{2}+T_{3} .
\end{aligned}
$$

Using the estimates (1.2), (2.12) and assumption (2.24), we have

$$
\begin{aligned}
\left\|T_{1}\right\|_{E} \leq & \lambda^{1-\alpha} \frac{1}{(k-1) !} \int_{0}^{\infty} t^{k-1} \exp (-t)\|A \exp \{-(\tau t+\lambda) A\} g(0)\|_{E} \\
\leq & \lambda^{1-\alpha} \frac{1}{(k-1) !} \int_{0}^{\infty} t^{k-1} \exp (-t) \frac{d t}{(t \tau+\lambda)^{1-\alpha}} \max _{-N \leq k \leq 0}\left\|g\left(t_{k}\right)\right\|_{E_{\alpha}} \\
\left\|T_{2}\right\|_{E} \leq & \lambda^{1-\alpha} \sum_{j=1}^{k} \tau \frac{1}{(k-j) !} \int_{0}^{\infty} t^{k-j} \exp (-t)\left\|A \exp \left\{-\frac{\tau t+\lambda}{2} A\right\}\right\|_{E \rightarrow E} \\
\leq & \frac{\lambda^{1-\alpha}(1-\alpha)(1-\epsilon)}{M 2^{2-\alpha}} \times B_{j} A^{-1}\left\|_{E \rightarrow E} \times\right\| A \exp \left\{-\frac{\tau t+\lambda}{2} A\right\} g\left(t_{j-N}\right) \|_{E} \\
& \times \sum_{j=1}^{k} \tau \frac{1}{(k-j) !} \int_{0}^{\infty} t^{k-j} \exp (-t) \frac{M 2^{2-\alpha} d t}{(t \tau+\lambda)^{2-\alpha}\left\|g\left(t_{j-N}\right)\right\|_{E_{\alpha}}} \\
\leq & \lambda^{1-\alpha}(1-\alpha)(1-\epsilon) \sum_{j=1}^{k} \tau \frac{1}{(k-j) !} \int_{0}^{\infty} t^{k-j} \exp (-t) \frac{d t}{(t \tau+\lambda)^{2-\alpha}} \\
& \times \max _{-N \leq k \leq 0}\left\|g\left(t_{k}\right)\right\| E_{\alpha}
\end{aligned}
$$


for every $k, 1 \leq k \leq N$ and $\lambda, \lambda>0$. Now we estimate $T_{3}$. By Lemma 2.3 and using the estimate (2.23), we obtain

$$
\begin{aligned}
\left\|T_{3}\right\|_{E} \leq & \lambda^{1-\alpha} \sum_{j=1}^{k} \tau \frac{1}{(k-j) !} \int_{0}^{\infty} t^{k-j} \exp (-t)\left\|A \exp \left\{-\frac{\tau t+\lambda}{2} A\right\}\right\|_{E \rightarrow E} \\
& \times\left\|A^{-1}\left[A \exp \left\{-\frac{\tau t+\lambda}{2} A\right\} B_{j}-B_{j} A \exp \left\{-\frac{\tau t+\lambda}{2} A\right\}\right] g\left(t_{j-N}\right)\right\|_{E} \\
\leq & \lambda^{1-\alpha} \max _{1 \leq k \leq N}\left\|\overline{A^{-1}\left(A B_{j}-B_{j} A\right) A^{-1}}\right\|_{E \rightarrow E} \sum_{j=1}^{k} \tau \frac{1}{(k-j) !} \\
& \times \int_{0}^{\infty} t^{k-j} \exp (-t) \frac{e(1+\alpha) M^{\alpha} M_{1}^{1+\alpha}\left(1+2 M_{1}\right)\left(1+M_{1}\right)^{1-\alpha} 2^{(2-\alpha) \alpha}}{\pi \alpha^{2}(1-\alpha)} \\
& \times \frac{M 2^{2-\alpha} d t}{(t \tau+\lambda)^{2-\alpha}}\left\|g\left(t_{j-N}\right)\right\|_{E_{\alpha}} \\
\leq & \lambda^{1-\alpha}(1-\alpha) \epsilon \sum_{j=1}^{k} \tau \frac{1}{(k-j) !} \int_{0}^{\infty} t^{k-j} \exp (-t) \frac{d t}{(t \tau+\lambda)^{2-\alpha}} \\
& \times \max _{-N \leq k \leq 0}\left\|g\left(t_{k}\right)\right\| E_{\alpha}
\end{aligned}
$$

for every $k, 1 \leq k \leq N$ and $\lambda, \lambda>0$. Using the triangle inequality we obtain

$$
\lambda^{1-\alpha}\left\|A \exp \{-\lambda A\} u_{k}\right\|_{E} \leq \max _{-N \leq k \leq 0} J\left\|g\left(t_{k}\right)\right\|_{E_{\alpha}}
$$

for every $k, 1 \leq k \leq N$ and $\lambda, \lambda>0$. This shows that

$$
\left\|u_{k}\right\|_{E_{\alpha}} \leq \max _{-N \leq k \leq 0}\left\|g\left(t_{k}\right)\right\|_{E_{\alpha}}
$$

for every $k, 1 \leq k \leq N$. Applying the mathematical induction, one can easily show that it is true for every $k$. Namely, assume that inequality (3.2) is true for every $k,(n-1) N \leq k \leq n N, n=1,2,3, \ldots$. Then for any $k, n N \leq k \leq(n+1) N$ using formula (2.8) we have 
284 Delay differential and difference equations

$$
\begin{aligned}
\lambda^{1-\alpha} A & \exp \{-\lambda A\} u_{k} \\
= & \lambda^{1-\alpha} \frac{1}{(k-n N-1) !} \int_{0}^{\infty} t^{k-n N-1} \exp (-t) A \exp \{-(\tau t+\lambda) A\} u_{n N} \\
& +\lambda^{1-\alpha} \sum_{j=n N+1}^{k} \tau \frac{1}{(k-j) !} \int_{0}^{\infty} t^{k-j} \exp (-t) \exp \left\{-\frac{\tau t+\lambda}{2} A\right\} B_{j} \\
& \times A \exp \left\{-\frac{\tau t+\lambda}{2} A\right\} u_{j-N} \\
& +\lambda^{1-\alpha} \sum_{j=n N+1}^{k} \frac{1}{(k-j) !} \int_{0}^{\infty} t^{k-j} \exp (-t) A \exp \left\{-\frac{\tau t+\lambda}{2} A\right\} \\
& \times A^{-1}\left[\exp \left\{-\frac{\tau t+\lambda}{2} A\right\} A B_{j}-B_{j} A \exp \left\{-\frac{\tau t+\lambda}{2} A\right\}\right] u_{j-N} \\
= & T_{1 n}+T_{2 n}+T_{3 n} .
\end{aligned}
$$

Using the estimates (1.2), (2.12), and assumption (2.24) we have

$$
\begin{aligned}
& \left\|T_{1 n}\right\|_{E} \leq \lambda^{1-\alpha} \frac{1}{(k-n N-1) !} \int_{0}^{\infty} t^{k-n N-1} \exp (-t)\left\|A \exp \{-(\tau t+\lambda) A\} u_{n N}\right\|_{E} \\
& \leq \lambda^{1-\alpha} \frac{1}{(k-n N-1) !} \int_{0}^{\infty} t^{k-n N-1} \exp (-t) \frac{d t}{(t \tau+\lambda)^{1-\alpha}} \max _{-N \leq k \leq 0}\left\|g\left(t_{k}\right)\right\|_{E_{\alpha}}, \\
& \left\|T_{2 n}\right\|_{E} \leq \lambda^{1-\alpha} \sum_{j=n N+1}^{k} \tau \frac{1}{(k-j) !} \int_{0}^{\infty} t^{k-j} \exp (-t)\left\|A \exp \left\{-\frac{\tau t+\lambda}{2} A\right\}\right\|_{E \rightarrow E} \\
& \times\left\|\overline{A^{-1} B_{j}}\right\|_{E \rightarrow E} \times\left\|A \exp \left\{-\frac{\tau t+\lambda}{2} A\right\} u_{j-N}\right\|_{E} \\
& \leq \frac{\lambda^{1-\alpha}(1-\alpha)(1-\epsilon)}{M 2^{2-\alpha}} \\
& \times \sum_{j=n N+1}^{k} \tau \frac{1}{(k-j) !} \int_{0}^{\infty} t^{k-j} \exp (-t) \frac{M 2^{2-\alpha} d t}{(t \tau+\lambda)^{2-\alpha}}\left\|u_{j-N}\right\|_{E_{\alpha}} \\
& \leq \lambda^{1-\alpha}(1-\alpha)(1-\epsilon) \sum_{j=n N+1}^{k} \tau \frac{1}{(k-j) !} \int_{0}^{\infty} t^{k-j} \exp (-t) \frac{d t}{(t \tau+\lambda)^{2-\alpha}} \\
& \times \max _{-N \leq k \leq 0}\left\|g\left(t_{k}\right)\right\|_{E_{\alpha}}
\end{aligned}
$$


for every $k, n N \leq k \leq(n+1) N, n=1,2,3, \ldots$, and $\lambda, \lambda>0$. Now we estimate $T_{3 n}$. By Lemma 2.3 and using the estimate (2.23), we obtain

$$
\begin{aligned}
\left\|T_{3 n}\right\|_{E} \leq & \lambda^{1-\alpha} \sum_{j=n N+1}^{k} \tau \frac{1}{(k-j) !} \int_{0}^{\infty} t^{k-j} \exp (-t)\left\|A \exp \left\{-\frac{\tau t+\lambda}{2} A\right\}\right\|_{E \rightarrow E} \\
& \times\left\|A^{-1}\left[A \exp \left\{-\frac{\tau t+\lambda}{2} A\right\} B_{j}-B_{j} A \exp \left\{-\frac{\tau t+\lambda}{2} A\right\}\right] u_{j-N}\right\|_{E} \\
\leq & \lambda^{1-\alpha} \max _{n N+1 \leq k \leq(n+1) N}\left\|\overline{A^{-1}\left(A B_{j}-B_{j} A\right) A^{-1}}\right\|_{E \rightarrow E} \sum_{j=1}^{k} \tau \frac{1}{(k-j) !} \\
& \times \int_{0}^{\infty} t^{k-j} \exp (-t) \frac{e(1+\alpha) M^{\alpha} M_{1}^{1+\alpha}\left(1+2 M_{1}\right)\left(1+M_{1}\right)^{1-\alpha} 2^{(2-\alpha) \alpha}}{\pi \alpha^{2}(1-\alpha)} \\
& \times \frac{M 2^{2-\alpha} d t}{(t \tau+\lambda)^{2-\alpha}\left\|u_{j-N}\right\|_{E_{\alpha}}} \\
\leq & \lambda^{1-\alpha}(1-\alpha) \epsilon \sum_{j=n N+1}^{k} \frac{1}{(k-j) !} \int_{0}^{\infty} t^{k-j} \exp (-t) \frac{d t}{(t \tau+\lambda)^{2-\alpha}} \\
& \times \max _{-N \leq k \leq 0}\left\|g\left(t_{k}\right)\right\| E_{\alpha}
\end{aligned}
$$

for every $k, n N \leq k \leq(n+1) N, n=1,2,3, \ldots$, and $\lambda, \lambda>0$. Using the triangle inequality we obtain

$$
\lambda^{1-\alpha}\left\|A \exp \{-\lambda A\} u_{k}\right\|_{E} \leq \max _{-N \leq k \leq 0} J_{n}\left\|g\left(t_{k}\right)\right\|_{E_{\alpha}}
$$

for every $k, n N \leq k \leq(n+1) N, n=1,2,3, \ldots$, and $\lambda, \lambda>0$. This shows that

$$
\left\|u_{k}\right\|_{E_{\alpha}} \leq \max _{-\omega \leq k \leq 0}\left\|g\left(t_{k}\right)\right\|_{E_{\alpha}}
$$

for every $k, n N \leq(n+1) N, n=1,2,3, \ldots$ This result completes the proof of Theorem 3.2.

Now we consider the difference scheme (3.2). We have not been able to obtain the same result for solution of the difference scheme (3.2) in the spaces $E_{\alpha}$ under the assumption for the difference scheme (3.1). But, we have the asymptotical stability of the solution of the difference scheme under the supplementary restriction of the operator $A$ that is considered in the following section. 
286 Delay differential and difference equations

\section{The difference scheme (3.2)}

THEOREM 4.1. Let condition (1.2) be satisfied. Suppose that the following estimate holds:

$$
\begin{gathered}
\left\|(I+\tau A)\left(I+\tau A+\frac{(\tau A)^{2}}{2}\right)^{-1}\right\|_{E \mapsto E} \leq 1, \\
\left\|\left(I+\frac{\tau A}{2}\right)(I+\tau A)\left(I+\tau A+\frac{(\tau A)^{2}}{2}\right)^{-1}\right\|_{E \mapsto E} \leq \frac{1+\sqrt{2}}{2},
\end{gathered}
$$

and

$$
\left\|B(t) A^{-1}\right\|_{E \mapsto E} \leq \frac{(1-\alpha)}{M 2^{1-\alpha}(1+\sqrt{2})}
$$

holds for every $t \geq 0$.

Then for the solution of difference scheme (3.2) the estimate

$$
\left\|u_{k}\right\|_{E_{\alpha}} \leq \max _{-N \leq k \leq 0}\left\|g\left(t_{k}\right)\right\|_{E_{\alpha}}
$$

holds for any $k \geq 1$.

Proof. Consider $1 \leq k \leq N$. In this case

$$
u_{k}=R^{k} g(0)+\sum_{j=1}^{k} R^{k-j+1}\left(I+\frac{\tau A}{2}\right)\left(g\left(t_{j-N}\right)+g\left(t_{j-N-1}\right)\right) \tau,
$$

where $R=\left(I+\tau A+(\tau A)^{2} / 2\right)^{-1}$. Using formula (3.5), the estimates (1.2), and assumption (4.1) we have

$$
\begin{aligned}
& \lambda^{1-\alpha}\left\|A \exp \{-\lambda A\} u_{k}\right\|_{E} \leq\left\|\left((I+\tau A)\left(I+\tau A+\frac{(\tau A)^{2}}{2}\right)^{-1}\right)^{k}\right\|_{E \mapsto E} \\
& \times \lambda^{1-\alpha} \frac{1}{(k-1) !} \int_{0}^{\infty} t^{k-1} \exp (-t)\|A \exp \{-(\tau t+\lambda) A\} g(0)\|_{E} \\
& +\lambda^{1-\alpha} \sum_{j=1}^{k} \tau\left\|\left((I+\tau A)\left(I+\tau A+\frac{(\tau A)^{2}}{2}\right)^{-1}\right)^{k-j}\right\|_{E \mapsto E} \\
& \quad \times\left\|\left(I+\frac{\tau A}{2}\right)(I+\tau A)\left(I+\tau A+\frac{(\tau A)^{2}}{2}\right)^{-1}\right\|_{E \mapsto E}
\end{aligned}
$$




$$
\begin{aligned}
& \times \frac{1}{(k-j) !} \int_{0}^{\infty} t^{k-j} \exp (-t)\left\|A \exp \left\{-\frac{\tau t+\lambda}{2} A\right\}\right\|_{E \rightarrow E}\left\|B_{j} A^{-1}\right\|_{E \rightarrow E} \\
& \times\left\|A \exp \left\{-\frac{\tau t+\lambda}{2} A\right\} \frac{1}{2}\left(g\left(t_{j-N}\right)+g\left(t_{j-N-1}\right)\right)\right\|_{E} \\
& \leq \lambda^{1-\alpha} \frac{1}{(k-1) !} \int_{0}^{\infty} t^{k-1} \exp (-t) \frac{d t}{(t \tau+\lambda)^{1-\alpha}}\|g(0)\|_{E_{\alpha}} \\
& +\frac{(1+\sqrt{2})}{2} \frac{1-\alpha}{M 2^{1-\alpha}(1+\sqrt{2})} \sum_{j=1}^{k} \tau \frac{1}{(k-j) !} \int_{0}^{\infty} t^{k-j} \exp (-t) \frac{M 2^{2-\alpha} d t}{(t \tau+\lambda)^{2-\alpha}} \\
& \quad \times \quad \frac{1}{2}\left(g\left(t_{j-N}\right)+g\left(t_{j-N-1}\right)\right) \|_{E_{\alpha}} \\
& \leq J \max _{-N \leq k \leq 0}\left\|g\left(t_{k}\right)\right\|_{E_{\alpha}} .
\end{aligned}
$$

Therefore

$$
\lambda^{1-\alpha}\left\|A \exp \{-\lambda A\} u_{k}\right\|_{E} \leq \max _{-N \leq k \leq 0}\left\|g\left(t_{k}\right)\right\|_{E_{\alpha}}
$$

for every $k, 1 \leq k \leq N$ and $\lambda, \lambda>0$. This shows that

$$
\left\|u_{k}\right\|_{E_{\alpha}} \leq \max _{-N \leq k \leq 0}\left\|g\left(t_{k}\right)\right\|_{E_{\alpha}}
$$

for every $k, 1 \leq k \leq N$. Assume that the estimate (4.3) is true for $k$, with $(n-1) N \leq k \leq n N, n=1,2,3, \ldots$. Then for any $k, n N \leq k \leq(n+1) N$ using formula (3.5) and

$$
u_{k}=R^{k-n N} u_{n N}+\sum_{j=n N+1}^{k} \tau R^{k-j}\left(I+\frac{\tau A}{2}\right) B_{j} \frac{1}{2}\left(u_{j-N}+u_{j-N-1}\right)
$$

the estimates (1.2), and assumption (4.1) we have that

$$
\begin{aligned}
\lambda^{1-\alpha} \| & A \exp \{-\lambda A\} u_{k} \|_{E} \\
\leq & \left\|\left((I+\tau A)\left(I+\tau A+\frac{(\tau A)^{2}}{2}\right)^{-1}\right)^{k-n N}\right\|_{E \mapsto E} \\
& \times \lambda^{1-\alpha} \frac{1}{(k-n N-1) !} \int_{0}^{\infty} t^{k-n N-1} \exp (-t)\left\|A \exp \{-(\tau t+\lambda) A\} u_{n N}\right\|_{E}
\end{aligned}
$$


Delay differential and difference equations

$$
\begin{aligned}
& +\lambda^{1-\alpha} \sum_{j=n N+1}^{k} \tau\left\|\left((I+\tau A)\left(I+\tau A+\frac{(\tau A)^{2}}{2}\right)^{-1}\right)^{k-j}\right\|_{E \mapsto E} \\
& \times\left\|\left(I+\frac{\tau A}{2}\right)(I+\tau A)\left(I+\tau A+\frac{(\tau A)^{2}}{2}\right)^{-1}\right\| \|_{E \mapsto E} \\
& \times \frac{1}{(k-j) !} \int_{0}^{\infty} t^{k-j} \exp (-t)\left\|A \exp \left\{-\frac{(\tau t+\lambda) A}{2}\right\}\right\|_{E \rightarrow E} \\
& \times\left\|B_{j} A^{-1}\right\|_{E \rightarrow E}\left\|A \exp \left\{-\frac{(\tau t+\lambda) A}{2}\right\} \frac{1}{2}\left(u_{j-N}+u_{j-N-1}\right)\right\|_{E} \\
& \leq \lambda^{1-\alpha} \frac{1}{(k-n N-1) !} \int_{0}^{\infty} t^{k-n N-1} \exp (-t) \frac{d t}{(t \tau+\lambda)^{1-\alpha}}\left\|u_{n N}\right\|_{E_{\alpha}} \\
& +\frac{(1+\sqrt{2})}{2} \frac{1-\alpha}{M 2^{1-\alpha}(1+\sqrt{2})} \sum_{j=n N+1}^{k} \tau \frac{1}{(k-j) !} \\
& \times \int_{0}^{\infty} t^{k-j} \exp (-t) \frac{M 2^{2-\alpha} d t}{(t \tau+\lambda)^{2-\alpha}}\left\|\frac{1}{2}\left(u_{j-N}+u_{j-N-1}\right)\right\|_{E_{\alpha}} \\
& \leq J_{n} \max _{(n-1) N \leq k \leq n N}\left\|u_{k}\right\|_{E_{\alpha}} .
\end{aligned}
$$

Therefore

$$
\lambda^{1-\alpha}\left\|A \exp \{-\lambda A\} u_{k}\right\|_{E} \leq \max _{-N \leq k \leq 0}\left\|g\left(t_{k}\right)\right\|_{E_{\alpha}}
$$

for every $k, n N \leq k \leq(n+1) N, n=1,2,3, \ldots$, and $\lambda, \lambda>0$. This shows that

$$
\left\|u_{k}\right\|_{E_{\alpha}} \leq \max _{-N \leq k \leq 0}\left\|g\left(t_{k}\right)\right\|_{E_{\alpha}}
$$

for every $k, n N \leq(n+1) N, n=1,2,3, \ldots$ This result completes the proof of Theorem 4.1.

Now, we consider the difference scheme (3.2) when

$$
A^{-1} B(t) x \neq B(t) A^{-1} x, \quad x \in D(A) .
$$

Suppose that

$$
\begin{aligned}
& \left\|\overline{A^{-1}(A B(t)-B(t) A) A^{-1}}\right\|_{E \mapsto E} \\
& \quad \leq \frac{\pi(1-\alpha)^{2} \alpha^{2}(1+\alpha)^{-1}(1+\sqrt{2})^{-1} \epsilon}{e M^{1+\alpha} M_{1}^{1+\alpha}\left(1+2 M_{1}\right)\left(1+M_{1}\right)^{1-\alpha} 2^{2+\alpha-\alpha^{2}}}
\end{aligned}
$$

holds for every $t \geq 0$. 
THEOREM 4.2. Let the assumptions (1.2) and (4.13) be satisfied. Suppose that the following estimate holds:

$$
\left\|\overline{A^{-1} B(t)}\right\|_{E \mapsto E} \leq \frac{(1-\alpha)(1-\epsilon)}{M 2^{2-\alpha}(1+\sqrt{2})}
$$

for every $t \geq 0$. Then for the solution of difference scheme (3.2) the estimate (4.3) holds for any $k \geq 1$.

Proof. Consider $1 \leq k \leq N$. Using formula (3.5) we obtain

$$
\begin{aligned}
& \lambda^{1-\alpha} A \exp \{-\lambda A\} u_{k} \\
& =\left((I+\tau A)\left(I+\tau A+\frac{(\tau A)^{2}}{2}\right)^{-1}\right)^{k} \\
& \times \lambda^{1-\alpha} \frac{1}{(k-1) !} \int_{0}^{\infty} t^{k-1} \exp (-t) A \exp \{-(\tau t+\lambda) A\} g(0) \\
& +\lambda^{1-\alpha} \sum_{j=1}^{k} \tau\left((I+\tau A)\left(I+\tau A+\frac{(\tau A)^{2}}{2}\right)^{-1}\right)^{k-j}\left(I+\frac{\tau A}{2}\right) \\
& \times(I+\tau A)\left(I+\tau A+\frac{(\tau A)^{2}}{2}\right)^{-1} \\
& \times \frac{1}{(k-j) !} \int_{0}^{\infty} t^{k-j} \exp (-t) \exp \left\{-\frac{(\tau t+\lambda) A}{2}\right\} \\
& \times B_{j} A \exp \left\{-\frac{(\tau t+\lambda) A}{2}\right\} \frac{1}{2}\left(g\left(t_{j-N}\right)+g\left(t_{j-N-1}\right)\right) \\
& +\lambda^{1-\alpha} \sum_{j=1}^{k} \tau\left((I+\tau A)\left(I+\tau A+\frac{(\tau A)^{2}}{2}\right)^{-1}\right)^{k-j}\left(I+\frac{\tau A}{2}\right) \\
& \times(I+\tau A)\left(I+\tau A+\frac{(\tau A)^{2}}{2}\right)^{-1} \\
& \times \frac{1}{(k-j) !} \int_{0}^{\infty} t^{k-j} \exp (-t) A \exp \left\{-\frac{(\tau t+\lambda) A}{2}\right\} A^{-1} \\
& \times\left[\exp \left\{-\frac{(\tau t+\lambda) A}{2}\right\} A B_{j}-B_{j} A \exp \left\{-\frac{(\tau t+\lambda) A}{2}\right\}\right] \\
& \times \frac{1}{2}\left(g\left(t_{j-N}\right)+g\left(t_{j-N-1}\right)\right) \\
& =P_{1}+P_{2}+P_{3} \text {. }
\end{aligned}
$$

Using the estimates (1.2), (2.12), and assumption (2.24), we have 
290 Delay differential and difference equations

$$
\begin{aligned}
& \left\|P_{1}\right\|_{E} \leq\left\|\left((I+\tau A)\left(I+\tau A+\frac{(\tau A)^{2}}{2}\right)^{-1}\right)^{k}\right\|_{E \mapsto E} \\
& \times \lambda^{1-\alpha} \frac{1}{(k-1) !} \int_{0}^{\infty} t^{k-1} \exp (-t)\|A \exp (-(\tau t+\lambda) A) g(0)\|_{E} \\
& \leq \lambda^{1-\alpha} \frac{1}{(k-1) !} \int_{0}^{\infty} t^{k-1} \exp (-t) \frac{d t}{(t \tau+\lambda)^{1-\alpha}} \max _{-N \leq k \leq 0}\left\|g\left(t_{k}\right)\right\|_{E_{\alpha}}, \\
& \left\|P_{2}\right\|_{E} \leq \lambda^{1-\alpha} \sum_{j=1}^{k} \tau\left\|\left((I+\tau A)\left(I+\tau A+\frac{(\tau A)^{2}}{2}\right)^{-1}\right)^{k-j}\right\|_{E \mapsto E} \\
& \times\left\|\left(I+\frac{\tau A}{2}\right)(I+\tau A)\left(I+\tau A+\frac{(\tau A)^{2}}{2}\right)^{-1}\right\|_{E \mapsto E} \\
& \times \frac{1}{(k-j) !} \int_{0}^{\infty} t^{k-j} \exp (-t)\left\|A \exp \left\{-\frac{(\tau t+\lambda) A}{2}\right\}\right\|_{E \rightarrow E} \\
& \times\left\|\overline{A^{-1} B_{j}}\right\|_{E \rightarrow E}\left\|A \exp \left\{-\frac{(\tau t+\lambda) A}{2}\right\} \frac{1}{2}\left(g\left(t_{j-N}\right)+g\left(t_{j-N-1}\right)\right)\right\|_{E} \\
& \leq \frac{(1+\sqrt{2})}{2} \frac{\lambda^{1-\alpha}(1-\alpha)(1-\epsilon)}{M 2^{1-\alpha}(1+\sqrt{2})} \\
& \times \sum_{j=1}^{k} \tau \frac{1}{(k-j) !} \int_{0}^{\infty} t^{k-j} \exp (-t) \frac{M 2^{2-\alpha} d t}{(t \tau+\lambda)^{2-\alpha}} \\
& \times\left\|\frac{1}{2}\left(g\left(t_{j-N}\right)+g\left(t_{j-N-1}\right)\right)\right\|_{E_{\alpha}} \\
& \leq(1-\alpha)(1-\epsilon) \sum_{j=1}^{k} \tau \frac{1}{(k-j) !} \int_{0}^{\infty} t^{k-j} \exp (-t) \frac{\lambda^{1-\alpha} d t}{(t \tau+\lambda)^{2-\alpha}} \\
& \times \max _{-N \leq k \leq 0}\left\|g\left(t_{k}\right)\right\|_{E_{\alpha}}
\end{aligned}
$$

for every $k, 1 \leq k \leq N$ and $\lambda, \lambda>0$. Now we estimate $P_{3}$. By Lemma 2.3 and using the estimate (2.23), we obtain

$$
\begin{aligned}
\left\|P_{3}\right\|_{E} \leq & \lambda^{1-\alpha} \sum_{j=1}^{k} \tau\left\|\left((I+\tau A)\left(I+\tau A+\frac{(\tau A)^{2}}{2}\right)^{-1}\right)^{k-j}\right\|_{E \mapsto E} \\
& \times\left\|\left(I+\frac{\tau A}{2}\right)(I+\tau A)\left(I+\tau A+\frac{(\tau A)^{2}}{2}\right)^{-1}\right\|_{E \mapsto E}
\end{aligned}
$$




$$
\begin{aligned}
& \times \frac{1}{(k-j) !} \int_{0}^{\infty} t^{k-j} \exp (-t)\left\|A \exp \left\{-\frac{(\tau t+\lambda) A}{2}\right\}\right\|_{E \rightarrow E} \\
& \times \| A^{-1}\left[A \exp \left\{-\frac{(\tau t+\lambda) A}{2}\right\} B_{j}-B_{j} A \exp \left\{-\frac{(\tau t+\lambda) A}{2}\right\}\right] \\
& \times \frac{1}{2}\left(g\left(t_{j-N}\right)+g\left(t_{j-N-1}\right)\right) \|_{E} \\
& \leq \frac{\lambda^{1-\alpha}(1+\sqrt{2})}{2} \max _{1 \leq j \leq N}\left\|\frac{A^{-1}\left(A B_{j}-B_{j} A\right) A^{-1}}{2}\right\|_{E \rightarrow E} \sum_{j=1}^{k} \tau \frac{1}{(k-j) !} \\
& \times \int_{0}^{\infty} t^{k-j} \exp (-t) \frac{e(1+\alpha) M^{\alpha} M_{1}^{1+\alpha}\left(1+2 M_{1}\right)\left(1+M_{1}\right)^{1-\alpha} 2^{(2-\alpha) \alpha}}{\pi \alpha^{2}(1-\alpha)} \\
& \quad \times \frac{M 2^{2-\alpha} d t}{(t \tau+\lambda)^{2-\alpha}} \frac{1}{2}\left\|\left(g\left(t_{j-N}\right)+g\left(t_{j-N-1}\right)\right)\right\|_{E_{\alpha}} \\
& \leq \lambda^{1-\alpha}(1-\alpha) \epsilon \sum_{j=1}^{k} \tau \frac{1}{(k-j) !} \int_{0}^{\infty} t^{k-j} \exp (-t) \frac{d t}{(t \tau+\lambda)^{2-\alpha}} \\
& \times \max _{-N \leq k \leq 0}\left\|g\left(t_{k}\right)\right\| E_{\alpha}
\end{aligned}
$$

for every $k, 1 \leq k \leq N$ and $\lambda, \lambda>0$. Using the triangle inequality we obtain

$$
\lambda^{1-\alpha}\left\|A \exp \{-\lambda A\} u_{k}\right\|_{E} \leq J \max _{-N \leq k \leq 0}\left\|g\left(t_{k}\right)\right\|_{E_{\alpha}}
$$

for every $k, 1 \leq k \leq N$ and $\lambda, \lambda>0$. This shows that

$$
\left\|u_{k}\right\|_{E_{\alpha}} \leq \max _{-N \leq k \leq 0}\left\|g\left(t_{k}\right)\right\|_{E_{\alpha}}
$$

for every $k, 1 \leq k \leq N$. Assume that inequality (3.3) is true for $k$, with ( $n-$ 1) $N \leq k \leq n N, n=1,2,3, \ldots$. Then for any $k, n N \leq k \leq(n+1) N$ using formula (2.8) we have that

$$
\begin{aligned}
\lambda^{1-\alpha} A \exp \{-\lambda A\} u_{k} \\
=\left((I+\tau A)\left(I+\tau A+\frac{(\tau A)^{2}}{2}\right)^{-1}\right)^{k-n N} \\
\quad \times \lambda^{1-\alpha} \frac{1}{(k-n N-1) !} \int_{0}^{\infty} t^{k-n N-1} \exp (-t) A \exp \{-(\tau t+\lambda) A\} u_{n N}
\end{aligned}
$$


Delay differential and difference equations

$$
\begin{aligned}
& +\lambda^{1-\alpha} \sum_{j=n N+1}^{k} \tau\left((I+\tau A)\left(I+\tau A+\frac{(\tau A)^{2}}{2}\right)^{-1}\right)^{k-j} \\
& \times\left(I+\frac{\tau A}{2}\right)(I+\tau A)\left(I+\tau A+\frac{(\tau A)^{2}}{2}\right)^{-1} \\
& \times \frac{1}{(k-j) !} \int_{0}^{\infty} t^{k-j} \exp (-t) \exp \left\{-\frac{(\tau t+\lambda) A}{2}\right\} B_{j} A \\
& +\lambda^{1-\alpha} \sum_{j=n N+1}^{k} \tau\left((I+\tau A)\left(I+\tau A+\frac{(\tau A)^{2}}{2}\right)^{-1}\right)^{k-j} \\
& \times\left(I+\frac{\tau A}{2}\right)(I+\tau A)\left(I+\tau A+\frac{(\tau A)^{2}}{2}\right)^{-1}\left\{\frac{(\tau t+\lambda) A}{2}\left(u_{j-N}+u_{j-N-1}\right)\right. \\
& \times \frac{1}{(k-j) !} \int_{0}^{\infty} t^{k-j} \exp (-t) A \exp \left\{-\frac{(\tau t+\lambda) A}{2}\right\} \\
& \times A^{-1}\left[\exp \left\{-\frac{(\tau t+\lambda) A}{2}\right\} A B_{j}-B_{j} A \exp \left\{-\frac{(\tau t+\lambda) A}{2}\right\}\right] \\
& \times \frac{1}{2}\left(u_{j-N}+u_{j-N-1}\right) \\
& =P_{1 n}+P_{2 n}+P_{3 n} .
\end{aligned}
$$

Using the estimates (1.2), (2.12), and assumption (2.24) we have

$$
\begin{aligned}
\left\|P_{1 n}\right\|_{E} \leq & \left\|\left((I+\tau A)\left(I+\tau A+\frac{(\tau A)^{2}}{2}\right)^{-1}\right)^{k-n N}\right\|_{E \mapsto E} \\
& \times \lambda^{1-\alpha} \frac{1}{(k-n N-1) !} \int_{0}^{\infty} t^{k-1} \exp (-t)\left\|A \exp \{-(\tau t+\lambda) A\} u_{n N}\right\|_{E} \\
\leq & \lambda^{1-\alpha} \frac{1}{(k-n N-1) !} \int_{0}^{\infty} t^{k-1} \exp (-t) \frac{d t}{(t \tau+\lambda)^{1-\alpha}} \max _{-N \leq k \leq 0}\left\|g\left(t_{k}\right)\right\|_{E_{\alpha}}, \\
\left\|P_{2 n}\right\|_{E} \leq & \lambda^{1-\alpha} \sum_{j=n N+1}^{k} \tau\left\|\left((I+\tau A)\left(I+\tau A+\frac{(\tau A)^{2}}{2}\right)^{-1}\right)^{k-j}\right\|_{E \mapsto E} \\
& \times\left\|\left(I+\frac{\tau A}{2}\right)(I+\tau A)\left(I+\tau A+\frac{(\tau A)^{2}}{2}\right)^{-1}\right\|
\end{aligned}
$$




$$
\begin{aligned}
& \times \frac{1}{(k-j) !} \int_{0}^{\infty} t^{k-j} \exp (-t)\left\|A \exp \left\{-\frac{(\tau t+\lambda) A}{2}\right\}\right\|_{E \rightarrow E} \\
& \times\left\|\overline{A^{-1} B_{j}}\right\|_{E \rightarrow E}\left\|A \exp \left\{-\frac{(\tau t+\lambda) A}{2}\right\} \frac{1}{2}\left(u_{j-N}+u_{j-N-1}\right)\right\|_{E} \\
\leq & \frac{(1+\sqrt{2})}{2} \frac{\lambda^{1-\alpha}(1-\alpha)(1-\epsilon)}{M 2^{1-\alpha}(1+\sqrt{2})} \\
& \times \sum_{j=n N+1}^{k} \tau \frac{1}{(k-j) !} \int_{0}^{\infty} t^{k-j} \exp (-t) \frac{M 2^{2-\alpha} d t}{(t \tau+\lambda)^{2-\alpha}} \\
& \times\left\|\frac{1}{2}\left(u_{j-N}+u_{j-N-1}\right)\right\|_{E_{\alpha}} \\
\leq & (1-\alpha)(1-\epsilon) \sum_{j=n N+1}^{k} \frac{1}{(k-j) !} \int_{0}^{\infty} t^{k-j} \exp (-t) \frac{\lambda^{1-\alpha} d t}{(t \tau+\lambda)^{2-\alpha}} \\
& \times \max _{-N \leq k \leq 0}\left\|g\left(t_{k}\right)\right\| E_{\alpha}
\end{aligned}
$$

for every $k, n N \leq k \leq(n+1) N, n=1,2,3, \ldots$, and $\lambda, \lambda>0$. Now we estimate $P_{3 n}$. By Lemma 2.3 and using the estimate (2.23), we obtain

$$
\begin{aligned}
\left\|P_{3 n}\right\|_{E} \leq & \lambda^{1-\alpha} \sum_{j=n N+1}^{k} \tau\left\|\left((I+\tau A)\left(I+\tau A+\frac{(\tau A)^{2}}{2}\right)^{-1}\right)^{k-j}\right\|_{E \mapsto E} \\
& \times\left\|\left(I+\frac{\tau A}{2}\right)(I+\tau A)\left(I+\tau A+\frac{(\tau A)^{2}}{2}\right)^{-1}\right\|_{E \mapsto E} \\
& \times \frac{1}{(k-j) !} \int_{0}^{\infty} t^{k-j} \exp (-t)\left\|A \exp \left\{-\frac{(\tau t+\lambda) A}{2}\right\}\right\|_{E \rightarrow E} \\
& \times \| A^{-1}\left[A \exp \left\{-\frac{(\tau t+\lambda) A}{2}\right\} B_{j}\right. \\
\leq & \frac{\lambda^{1-\alpha}(1+\sqrt{2})}{2} \max _{n N+1 \leq j \leq(n+1) N}\left\|\overline{A^{-1}\left(A B_{j}-B_{j} A\right) A^{-1}}\right\|_{E \rightarrow E} \\
& \times \sum_{j=n N+1}^{k} \frac{1}{(k-j) !}
\end{aligned}
$$




$$
\begin{aligned}
& \quad \times \int_{0}^{\infty} t^{k-j} \exp (-t) \frac{e(1+\alpha) M^{\alpha} M_{1}^{1+\alpha}\left(1+2 M_{1}\right)\left(1+M_{1}\right)^{1-\alpha} 2^{(2-\alpha) \alpha}}{\pi \alpha^{2}(1-\alpha)} \\
& \quad \times \frac{M 2^{2-\alpha} d t}{(t \tau+\lambda)^{2-\alpha}} \frac{1}{2}\left\|u_{j-N}+u_{j-N-1}\right\|_{E_{\alpha}} \\
& \leq \lambda^{1-\alpha}(1-\alpha) \epsilon \sum_{j=n N+1}^{k} \tau \frac{1}{(k-j) !} \\
& \quad \times \int_{0}^{\infty} t^{k-j} \exp (-t) \frac{d t}{(t \tau+\lambda)^{2-\alpha}} \max _{-N \leq k \leq 0}\left\|g\left(t_{k}\right)\right\|_{E_{\alpha}}
\end{aligned}
$$

for every $k, n N \leq k \leq(n+1) N$ and $\lambda, \lambda>0$. Using the triangle inequality we obtain

$$
\lambda^{1-\alpha}\left\|A \exp \{-\lambda A\} u_{k}\right\|_{E} \leq J_{n} \max _{-N \leq k \leq 0}\left\|g\left(t_{k}\right)\right\|_{E_{\alpha}}
$$

for every $k, n N \leq k \leq(n+1) N, n=1,2,3, \ldots$, and $\lambda, \lambda>0$. This shows that

$$
\left\|u_{k}\right\|_{E_{\alpha}} \leq \max _{-\omega \leq k \leq 0}\left\|g\left(t_{k}\right)\right\|_{E_{\alpha}}
$$

for every $k, n N \leq(n+1) N, n=1,2,3, \ldots$ This result completes the proof of Theorem 4.2.

\section{Applications}

We consider the initial-value problem on the range $\left\{0 \leq t \leq 1, x \in \mathbb{R}^{n}\right\}$ for $2 m$-order multidimensional delay differential equations of parabolic type

$$
\begin{aligned}
& \frac{\partial u(t, x)}{\partial t}+\sum_{|\tau|=2 m} a_{\tau}(x) \frac{\partial^{|\tau|} u(t, x)}{\partial x_{1}^{\tau_{1}} \cdots \partial x_{n}^{\tau_{n}}}+\delta u(t, x) \\
& \quad=b(t)\left(\sum_{|\tau|=2 m} a_{\tau}(x) \frac{\partial^{|\tau|} u(t-\omega, x)}{\partial x_{1}^{\tau_{1}} \cdots \partial x_{n}^{\tau_{n}}}+\delta u(t-\omega, x)\right), \quad 0<t<\infty, \quad x \in \mathbb{R}^{n}, \\
& u(t, x)=g(t, x), \quad-\omega \leq t \leq 0, x \in \mathbb{R}^{n}, \quad|\tau|=\tau_{1}+\cdots+\tau_{n},
\end{aligned}
$$

where $a_{r}(x), b(t), g(t, x)$ are given sufficiently smooth functions and $\delta>0$ is the sufficiently large number. We will assume that the symbol

$$
A_{1}^{x}(\xi)=\sum_{|\tau|=2 m} a_{r}(x)(i \xi)^{r_{1}} \cdots(i \xi)^{r_{n}}
$$

of the differential operator of the form

$$
A_{1}^{x}=\sum_{|r=2 m|} a_{r}(x) \frac{\partial^{|r|}}{\partial x_{1}^{r_{1}} \cdots \partial x_{n}^{r_{n}}}
$$


acting on functions defined on the space $\mathbb{R}^{n}$, satisfies the inequalities

$$
0<M_{1}|\xi|^{2 m} \leq\left|A_{1}^{x}(\xi)\right| \leq M_{2}|\xi|^{2 m}<\infty \text { for } \xi \neq 0 .
$$

Problem (5.1) has a unique smooth solution. This allows us to reduce the initial-value problem (5.1) to the initial-value problem (1.1) in the Banach space $E$ with a strongly positive operator $A^{x}=A_{1}^{x}+\delta I$ defined by (5.4). We give a number of corollaries of the abstract Theorems 2.1 and 3.1.

THEOREM 5.1. Assume that

$$
\sup _{0 \leq t<\infty}|b(t)| \leq \frac{1-\alpha}{M 2^{2-\alpha}} .
$$

Then the solutions of the initial-value problem (5.1) satisfy the following stability estimates:

$$
\sup _{0 \leq t<\infty}\|v(t, \cdot)\|_{C^{2 m \alpha}\left(\mathbb{R}^{n}\right)} \leq M_{2}(\alpha) \max _{-\omega \leq t \leq 0}\|g(t, \cdot)\|_{C^{2 m \alpha}\left(\mathbb{R}^{n}\right)}, \quad 0<\alpha<\frac{1}{2 m},
$$

where $M_{2}(\alpha)$ does not depend on $g(t, x)$. Here $C^{\varepsilon}\left(\mathbb{R}^{n}\right)$ is the space of functions satisfying a Hölder condition with the indicator $\varepsilon \in(0,1)$.

The proof of Theorem 5.1 is based on the estimate

$$
\left\|\exp \left\{-t A^{x}\right\}\right\|_{C\left(\mathbb{R}^{n}\right) \rightarrow C\left(\mathbb{R}^{n}\right)} \leq M, \quad t \geq 0,
$$

and on the abstract Theorem 2.1, on the strong positivity of the operator $A^{x}$ in $C^{\varepsilon}\left(\mathbb{R}^{n}\right)$, and on the fact of the equivalence of the norms in the spaces $E_{\alpha}=$ $E_{\alpha}\left(A, C\left(\mathbb{R}^{n}\right)\right)$ and $C^{2 m \alpha}\left(\mathbb{R}^{n}\right)$ when $0<\alpha<1 / 2 m$ (see [5]).

We define the grid space $\mathbb{R}_{h}^{n}\left(0<h \leq h_{0}\right)$ as the set of all points of the Euclidean space $\mathbb{R}^{n}$ whose coordinates are given by

$$
x_{i}=s_{i} h, \quad s_{i}=0, \pm 1, \pm 2, \ldots, \quad i=1, \ldots, n .
$$

To the differential operator $A^{x}=A_{1}^{x}+\delta I$ defined by (5.4) we assign the difference operator $A_{h}^{x}$ by the formula

$$
A_{h}^{x} u^{h}(x)=\sum_{2 m \leq|r| \leq S} b_{r}^{h}(x) D_{h}^{r} u^{h}(x)
$$

of an arbitrary high order of accuracy that approximates this multidimensional elliptic operator $A^{x}$ which acts on functions $u_{x}^{h}$ defined on the entire space $\mathbb{R}_{h}^{n}$. We will assume that for $\left|\xi_{k} h\right| \leq \pi$, the symbol $A^{x}(\xi h, h)$ of the operator $A_{h}^{x}$ satisfies the inequalities

$$
\left|A^{x}(\xi h, h)\right| \geq M_{3}|\xi|^{2 m}, \quad\left|\arg A^{x}(\xi h, h)\right| \leq \phi<\pi .
$$


With the help of $A_{h}^{x}$ we arrive at the initial-value problem

$$
\begin{gathered}
\left(u^{h}(t, x)\right)^{\prime}+A_{h}^{x} u^{h}(t, x)=b(t) A_{h}^{x} u^{h}(t-\omega, x), \quad t \geq 0, x \in \mathbb{R}^{n}, \\
u^{h}(t, x)=g^{h}(t, x)=g(t, x) \quad(-\omega \leq t \leq 0), x \in \mathbb{R}^{n},
\end{gathered}
$$

for an infinite system of ordinary differential equations. Now, we replace problem (5.12) by the first order accuracy in $t$ difference scheme

$$
\begin{gathered}
\frac{1}{\tau}\left(u_{k}^{h}(x)-u_{k-1}^{h}(x)\right)+A_{h}^{x} u_{k}^{h}(x)=b\left(t_{k}\right) A_{h}^{x} u_{k-N}^{h}(x), \\
t_{k}=k \tau, 1 \leq k, N \tau=\omega, x \in \mathbb{R}_{h}^{n}, \\
u_{k}^{h}(x)=g^{h}\left(t_{k}, x\right), \quad t_{k}=k \tau,-N \leq k \leq 0, x \in \mathbb{R}_{h}^{n} .
\end{gathered}
$$

THEOREM 5.2. Assume that all the conditions of Theorem 5.1 are satisfied. Then for the solution of difference scheme (5.13) the estimate

$$
\sup _{1 \leq k<\infty}\left\|u_{k}^{h}\right\|_{C^{2 m \alpha}\left(\mathbb{R}_{h}^{n}\right)} \leq M_{2}(\alpha) \max _{-N \leq t \leq 0}\left\|g_{k}^{h}\right\|_{C^{2 m \alpha}\left(\mathbb{R}_{h}^{n}\right)}, \quad 0<\alpha<\frac{1}{2 m},
$$

holds.

The proof of Theorem 5.2 is based on the estimate

$$
\left\|\exp \left\{-t_{k} A_{h}^{x}\right\}\right\|_{C\left(\mathbb{R}_{h}^{n}\right) \rightarrow C\left(\mathbb{R}_{h}^{n}\right)} \leq M, \quad k \geq 0,
$$

and on the abstract Theorem 3.1 , the positivity of the operator $A_{h}^{x}$ in $C^{\varepsilon}\left(\mathbb{R}_{h}^{n}\right)$, and on the fact that the $E_{\alpha}=E_{\alpha}\left(A_{h}^{x}, C\left(\mathbb{R}_{h}^{n}\right)\right)$-norms are equivalent to the norms $C^{2 m \alpha}\left(\mathbb{R}_{h}^{n}\right)$ uniformly in $h$ for $0<\alpha<1 / 2 m$ (see [5]).

\section{References}

[1] A. N. Al-Mutib, Stability properties of numerical methods for solving delay differential equations, J. Comput. Appl. Math. 10 (1984), no. 1, 71-79. MR 85m:65075. Zbl 542.65040.

[2] A. Ashyralyev and H. Akça, On difference schemes for semilinear delay differential equations with constant delay, Actual Problems of Applied Mathematics, Physics and Engineering (Ashgabad), 1999, pp. 18-21.

[3] - Stability estimates of difference schemes for neutral delay differential equations, Nonlinear Anal. 44 (2001), no. 4, 443-452. CMP 1822094.

[4] A. Ashyralyev, H. Akça, and U. Guray, Second order accuracy difference scheme for approximate solutions of delay differential equations, Funct. Differ. Equ. 6 (1999), no. 3-4, 223-231. CMP 1809191.

[5] A. Ashyralyev and P. E. Sobolevskii, Well-Posedness of Parabolic Difference Equations, Operator Theory: Advances and Applications, vol. 69, Birkhäuser Verlag, Basel, 1994. MR 95j:65094.

[6] A. Bellen, One-step collocation for delay differential equations, J. Comput. Appl. Math. 10 (1984), no. 3, 275-283. MR 85m:65061. Zbl 538.65047. 
[7] A. Bellen, Z. Jackiewicz, and M. Zennaro, Stability analysis of one-step methods for neutral delay-differential equations, Numer. Math. 52 (1988), no. 6, 605-619. MR 89h:65134. Zbl 644.65049.

[8] A. Bellen and M. Zennaro, Strong contractivity properties of numerical methods for ordinary and delay differential equations, Appl. Numer. Math. 9 (1992), no. 3-5, 321-346, from ASU conference 1990. MR 93a:65104. Zbl 0749.65042.

[9] K. L. Cooke and I. Györi, Numerical approximation of the solutions of delay differential equations on an infinite interval using piecewise constant arguments, Comput. Math. Appl. 28 (1994), no. 1-3, 81-92. MR 95f:65141. Zbl 809.65074.

[10] T. Hong-Jiong and K. Jiao-Xun, The numerical stability of linear multistep methods for delay differential equations with many delays, SIAM J. Numer. Anal. 33 (1996), no. 3, 883-889. MR 97d:65037. Zbl 855.65094.

[11] M. A. Krasnoselskiŭ, P. P. Zabrě̌ko, E. I. Pustylnik, and P. E. Sobolevskii, Integral Operators in Spaces of Summable Functions, Monographs and Textbooks on Mechanics of Solids and Fluids, Mechanics: Analysis, Noordhoff, Leiden, 1976. MR 52\#6505.

[12] L. Lianhua, Numerical stability of the $\theta$-methods for systems of differential equations with several delay terms, J. Comput. Math. Appl. 34 (1991), 291-304.

[13] 101-114.

[14] M. Z. Liu and M. N. Spijker, The stability of the $\theta$-methods in the numerical solution of delay differential equations, IMA J. Numer. Anal. 10 (1990), no. 1, 31-48. MR 91e:65106. Zbl 693.65056.

[15] L. Torelli, Stability of numerical methods for delay differential equations, J. Comput. Appl. Math. 25 (1989), no. 1, 15-26. MR 90f:65146. Zbl 664.65073.

[16] - A sufficient condition for GPN-stability for delay differential equations, Numer. Math. 59 (1991), no. 3, 311-320. MR 92h:65127. Zbl 726.65106.

[17] H. Triebel, Interpolation Theory, Function Spaces, Differential Operators, NorthHolland Mathematical Library, vol. 18, North-Holland, Amsterdam, 1978. MR 80i:46032b. Zbl 387.46032.

A. Ashyralyev: Department of Mathematics, Fatih University, 34900, BuyukCEKMECE, Istanbul, TuRKey

Current address: Department of Mathematics, International Turkmen-Turkish

University, 84, Gerogly, 744012, Ashgabat, Turkmenistan

E-mail address: aashyr@fatih.edu.tr

P. SobolevskiI: Institute of Mathematics, Hebrew University, Jerusalem, ISRAEL

E-mail address: pavels@math.hiji.ae.il 


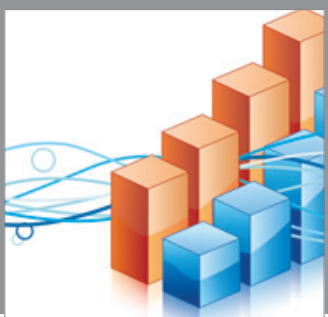

Advances in

Operations Research

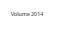

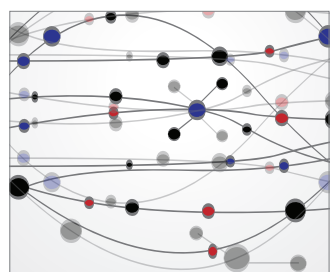

\section{The Scientific} World Journal
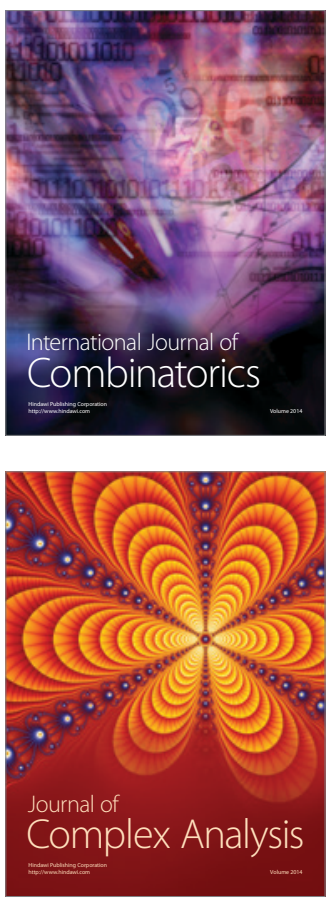

International Journal of

Mathematics and

Mathematical

Sciences
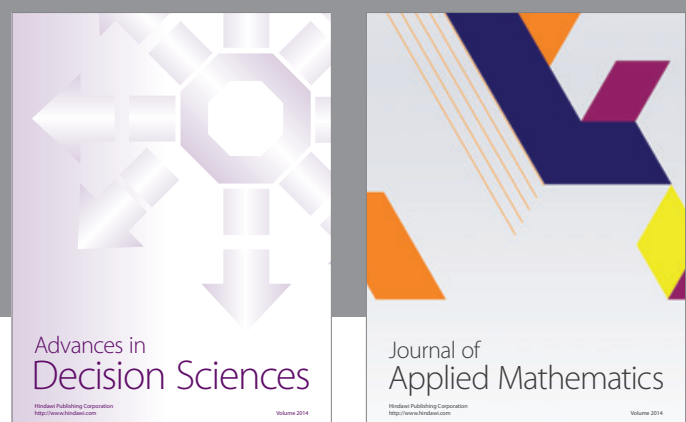

Journal of

Applied Mathematics
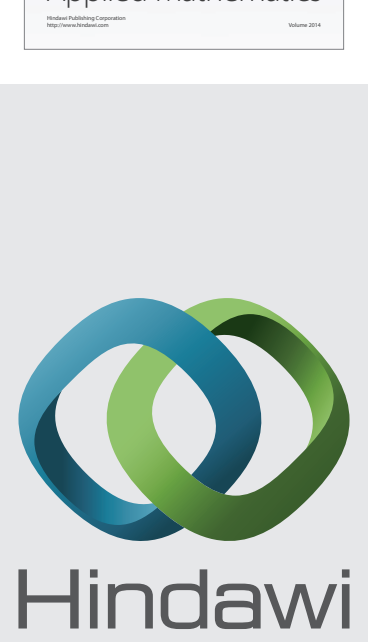

Submit your manuscripts at http://www.hindawi.com
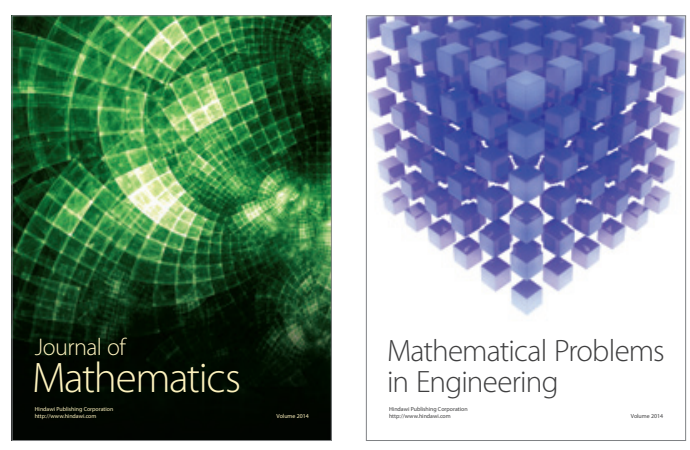

Mathematical Problems in Engineering
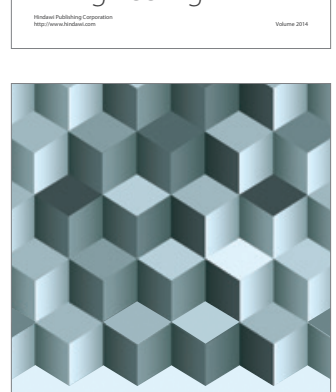

Journal of

Function Spaces
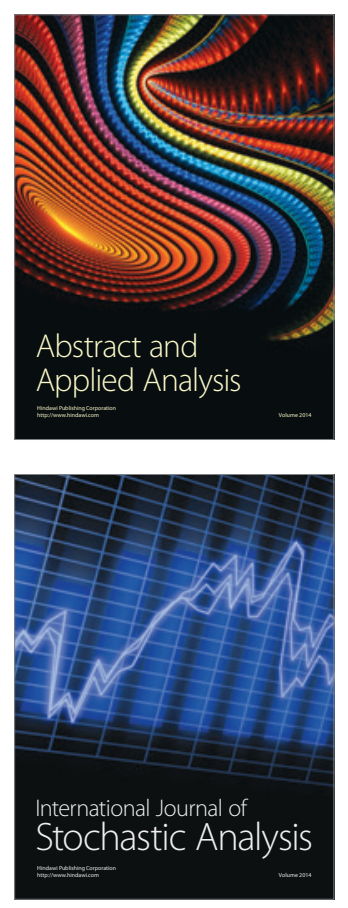

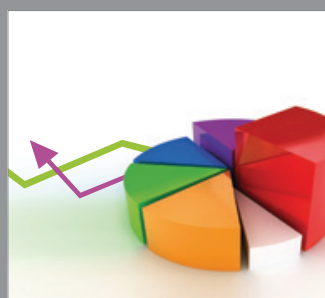

ournal of

Probability and Statistics

Promensencen
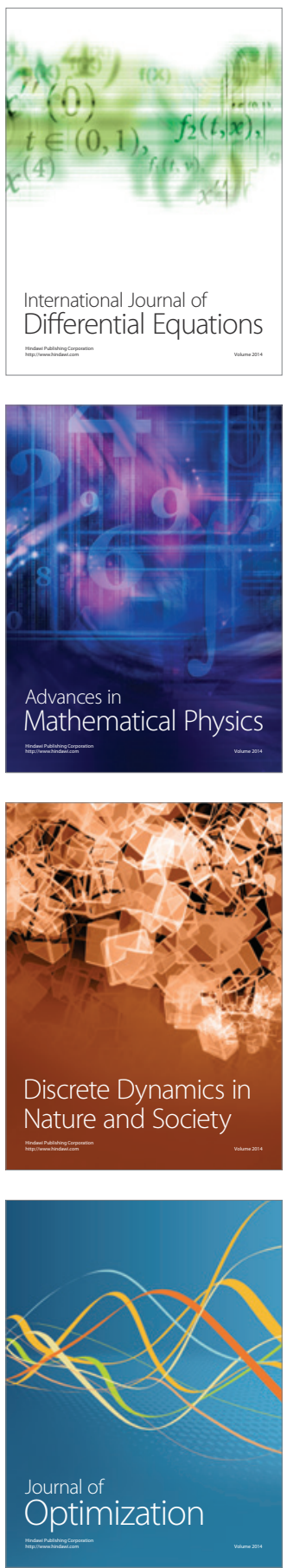